\title{
Medical physics aspects of the synchrotron radiation therapies: Microbeam radiation therapy (MRT) and synchrotron stereotactic radiotherapy (SSRT)
} \author{
Erik A. Siegbahn ${ }^{\mathrm{m}}$, Dan Sporea ${ }^{\mathrm{n}}$, Bjarne Stugu ${ }^{\circ}$ \\ ${ }^{a}$ ESRF-The European Synchrotron, 71, Avenue des Martyrs, Grenoble, France \\ b INSERM, Grenoble, France \\ ${ }^{c}$ University of Bergen Department of Physics and Technology, PB 7803 5020, Norway \\ ${ }^{\mathrm{d}}$ The Institute of Cancer Research, 15 Cotswold Rd, Sutton SM2 5NG, United Kingdom \\ e RMIT University, Melbourne, VIC, 3001, Australia \\ ${ }^{\mathrm{f}}$ Ghent University Hospital, 9000 Gent, Belgium \\ ${ }^{g}$ Centre for Medical Radiation Physics, University of Wollongong, Northfields Ave, NSW, Australia \\ ${ }^{\mathrm{h}}$ CRUK Cancer Imaging Centre, Institute of Cancer Research, 15 Cotswold Rd, Sutton Surrey, UK \\ i Medical Physics Laboratory, University of Ioannina, 451.10, Ioannina, Greece \\ j Sintef Minalab, Gaustadalléen 23C, 0373, Oslo, Norway \\ ${ }^{\mathrm{k}}$ Institute of Nuclear Physics PAN, Radzikowskiego 152, 31-342, Krawkow, Poland \\ ${ }^{1}$ University of Oslo, Department of Physics, 0316, Oslo, Norway \\ m Department of Oncolgy-Pathology, Karolinska Institutet, S-177176, Stockholm, Sweden \\ ${ }^{\mathrm{n}}$ National Institute for Laser, Plasma and Radiation Physics, Magurele, RO-077125, Romania \\ ${ }^{\circ}$ University of Bergen, Department of Physics and Technology, PB 7803, 5020, Bergen, Norway
}

Elke Bräuer-Krisch ${ }^{\text {a, }}$, Jean-Francois Adam ${ }^{\text {b }}$, Enver Alagoz ${ }^{c}$, Stefan Bartzsch ${ }^{\mathrm{d}}$, Jeff Crosbie ${ }^{\mathrm{e}}$, Carlos DeWagter ${ }^{\mathrm{f}}$, Andrew Dipuglia ${ }^{\mathrm{g}}$, Mattia Donzelli ${ }^{\mathrm{a}}$, Simon Doran ${ }^{\mathrm{h}}$, Pauline Fournier ${ }^{\mathrm{a}, \mathrm{g}}$, John Kalef-Ezra ${ }^{\mathrm{i}}$, Angela Kock ${ }^{\mathrm{j}}$, Michael Lerch ${ }^{\mathrm{g}}$, Ciara McErlean ${ }^{\mathrm{h}}$, Uwe Oelfke ${ }^{\mathrm{d}}$, Pawel Olko ${ }^{\mathrm{k}}$, Marco Petasecca ${ }^{\mathrm{g}}$, Marco Povoli ${ }^{\mathrm{l}}$, Anatoly Rosenfeld ${ }^{\mathrm{g}}$,

\section{A R T I C L E I N F O}

\section{Article history:}

Received 22 December 2014

Received in revised form

27 April 2015

Accepted 28 April 2015

Available online 1 June 2015

\section{Keywords}

Microbeam radiation therapy

SSRT

Monte Carlo calculations

MRT

Radiation oncology

Synchrotron X-rays

Dosimetry

\begin{abstract}
A B S T R A C T
Stereotactic Synchrotron Radiotherapy (SSRT) and Microbeam Radiation Therapy (MRT) are both novel approaches to treat brain tumor and potentially other tumors using synchrotron radiation. Although the techniques differ by their principles, SSRT and MRT share certain common aspects with the possibility of combining their advantages in the future. For MRT, the technique uses highly collimated, quasi-parallel arrays of X-ray microbeams between 50 and $600 \mathrm{keV}$. Important features of highly brilliant Synchrotron sources are a very small beam divergence and an extremely high dose rate. The minimal beam divergence allows the insertion of so called Multi Slit Collimators (MSC) to produce spatially fractionated beams of typically 25-75 micron-wide microplanar beams separated by wider (100-400 microns center-to-center(ctc)) spaces with a very sharp penumbra. Peak entrance doses of several hundreds of Gy are extremely well tolerated by normal tissues and at the same time provide a higher therapeutic index for various tumor models in rodents. The hypothesis of a selective radio-vulnerability of the tumor vasculature versus normal blood vessels by MRT was recently more solidified.

SSRT (Synchrotron Stereotactic Radiotherapy) is based on a local drug uptake of high-Z elements in tumors followed by stereotactic irradiation with $80 \mathrm{keV}$ photons to enhance the dose deposition only within the tumor. With SSRT already in its clinical trial stage at the ESRF, most medical physics problems are already solved and the implemented solutions are briefly described, while the medical physics aspects in MRT will be discussed in more detail in this paper.

(c) 2015 Published by Elsevier Ltd on behalf of Associazione Italiana di Fisica Medica. This is an open access article under the CC BY-NC-ND license (http://creativecommons.org/licenses/by-nc-nd/4.0/).
\end{abstract}

\footnotetext{
* Corresponding author. Tel.: +33 (0) 476882115; fax: +33 (0) 476882020.

E-mail address: brauer@esrf.fr (E. Bräuer-Krisch).
} 


\section{Introduction}

The different contributions within the COST SYRA3 Action in this special issue highlight the history of the development of two new radiotherapies; MRT and SSRT, and their future potential medical applications. The phase I clinical trials in SSRT have allowed the community to move forward with synchrotron based therapies in particular from a safety point of view, requiring the implementation of a small hospital-like environment at the biomedical beamline ID17 at the European Synchrotron Radiation Facility (ESRF) in Grenoble, France. This milestone also helps to solve some of the medical physics aspects in MRT which are particularly challenging due to the microscopically small sizes of the beams and the very high dose rates. This high dose gradient requires accurate measurements of dose in microscopic volumes, something which is not necessary in standard radiotherapy. Despite the increasing computing power, Monte Carlo (MC) calculations in such small volumes for MRT applications are still time consuming and a recently developed solution using a convolution based algorithm now allows fast dose calculations from $\mathrm{CT}$ data to make a treatment plan. Dose measurements in MRT are difficult not only due to the demands on the spatial resolution but equally from the high dose rates used which are in the range of $8-16 \mathrm{kGy} / \mathrm{s}$. Additionally, the low energy photons may require an important correction since the response of commonly used radiation detectors shows important variations for low energy X-ray photons.

\section{Medical physics aspects in SSRT}

The first clinical study of therapeutic applications of ContrastEnhanced Synchrotron Stereotactic Radiation Therapy (SSRT) has been underway since June 2012 at the (ESRF) and at the University Hospital (CHU) in Grenoble (France). This phase I-II clinical trial is designed to test the feasibility and safety of SSRT through a dose escalation protocol. Two years after the start of the trial, this study has already included eight patients suffering from brain metastases of medium-to-small volume. Preclinical studies [1,2], based on the original work of Norman [3] had highlighted the potential of the technique and motivated this clinical trial. The treatment at the ESRF is based on stereotactic irradiations using high-flux, quasi-parallel, monochromatic medium energy X-ray beams ( $80 \mathrm{keV})$. The irradiation is performed, in the presence of an iodinated contrast agent, which previously was introduced into the tumor. At these energies, a localized dose enhancement occurs in the target, due to an increased photoelectric absorption of X-rays. This local increase in dose is due to the difference in the photon interaction mechanisms in the target volume where the contrast agent leaks from the capillaries when compared to the healthy brain where the iodine concentration remains negligible. The moderate kinetic energy of the photoelectrons and the iodine Auger electrons is deposited over a micrometer distance with a maximum distance of tens of micros, in the close vicinity of the heavy atoms; whereas Compton scattering predominates in the surrounding healthy tissues. Despite a strong falloff of the percentage depth dose (PDD) using $80 \mathrm{keV}$ photons, a favorable dose deposition can be achieved at the tumor with better tissue sparing when compared to Co-60 irradiations using the same number of ports, thus generating interest for treating deep seated tumors.

A dedicated treatment room has been built at the ESRF medical beamline [4]. The patient is installed on an armchair with his or her head tightly maintained by the same stereotactic frame used at the CHU for complimentary irradiations. The current dosimetry protocol in SSRT uses monochromatic X-rays at $80 \mathrm{keV}$ with a dose rate of $\sim 1 \mathrm{~Gy} / \mathrm{s}$ which is slightly higher but in the same order of magnitude like typical dose rates at the clinic. The specificity comes from the use of a $2 \mathrm{~mm}$ high beam, requiring the regular scanning through the beam to obtain a homogenous coverage of the tumor volume to be irradiated. A dedicated treatment planning system (TPS) was adapted to SSRT. The synchrotron beamline geometry was modeled and included as a phase space file in the TPS. The dosimetry is based on parallelized Monte Carlo simulations of low to medium energy electrons and polarized photon transport in presence of high-Z material [5]. Dedicated quality assurance protocols were implemented. An absolute dosimetry protocol was adapted according to the gold standard used in conventional RT [6]. The treatment plans and absolute dosimetry are validated with measurements performed in a dedicated water tank as well as in solid water with and without bone slabs. A 2D dosimetry technique is being developed in anthropomorphic phantoms using EBT3 Gafchromic films.

The contrast agent uptake has been previously studied on 12 patients who received an intravenous bolus of iodinated contrast agent $(40 \mathrm{~mL}, 4 \mathrm{~mL} / \mathrm{s})$, followed by a steady-state infusion $(160 \mathrm{~mL}$, $0.5 \mathrm{~mL} / \mathrm{s}$ ) in order to ensure stable intratumoral amounts of iodine during the treatment. Absolute iodine concentrations and quantitative perfusion maps were derived from 40 multi-slice dynamic conventional CT images of the brain (recruitment day) or from quantitative synchrotron radiation CT (treatment day). For three of these patients, iodine concentrations reached in the tumor were compared between the recruitment day and the treatment day $(\sim 10$ days interval). The post-infusion mean intratumoral iodine concentration (over $30 \mathrm{~min}$ ) reached $1.94 \pm 0.12 \mathrm{mg} / \mathrm{mL}(200 \mathrm{~mL}$ of contrast injected) [7].

In this first clinical trial phase, the patients receive a fraction of their overall treatment by SSRT ( $5 \mathrm{~Gy}$ ), while the remaining of the treatment is delivered by standard stereotactic irradiation at the CHU (6 Gy and $2 \times 11 \mathrm{~Gy}$ ). All patients were in good general condition [8]. Future developments in medical physics for SSRT are expected to include in invivo dosimetry and static irradiations using minibeams [9].

In vivo dosimetry based on optically stimulated luminescence $\left(\mathrm{Al}_{2} \mathrm{O}_{3}\right.$ crystals) has already been tested on one patient [10] but requires a complex set-up and offline reading. A new in vivo dosimetry protocol is currently being developed, based on 2D entrance and exit fluence measurements using dedicated pixelated transmission detectors. The dose retrieval will be performed using inverse problem methods (iterative reconstruction of the dose) adapted to local and limited projection tomography problems [11]. The monochromatic minibeam technique is being developed in parallel to further improve the normal tissue sparing effect and simplify some of the delicate safety issues because of the lower dose rate. The first experiments in monochromatic Minibeam Radiation Therapy (MBRT) (600 $\mu \mathrm{m}$-wide beams, $1200 \mu \mathrm{m}$ ctc) confirmed that this technique keeps (part of) the sparing tissue capability observed in the thinner microbeams, while significant tumor growth delay was still observed [12]. The next development is the transfer of this technique to clinical trials I in order to be able to perform the SSRT dose escalation protocol to its end maintaining a suitable bone radiation tolerance [13].

The non-homogenous dose distribution due to the irregular uptake in the tumor environment of the dose enhancing drug leads to in-homogeneities, which may complicate the interpretation of the outcome of the treatment [7]. The contrast agent, moreover, remains extracellular and is not optimal for dose enhancement at the cellular and molecular levels with respect to the DNA. An interesting perspective would be to influence importantly the microscopic dose distribution from Auger electrons through photon activation processes [14] or from optimized radio-chemotherapy protocols [15], which would more selectively damage the tumor cells with non-repairable double strand breaks. 
The simulations for such processes are often lacking the detailed inputs relating to knowledge about the microscopic drug distribution to be assumed. This may be another important avenue to be fostered within the current COST SYRA3 action to improve the local dose deposition within the tumor cell. Such dose enhancement should be better understood and warrant microdosimetry studies. Adequate Monte Carlo calculations are used to predict such local dose distributions and cover an entire research field on its own. However, results from such research efforts could be best exploited and tested on the ID17 biomedical beamline through preclinical studies using tunable monochromatic X-rays, before being transferred to clincial trials.

\section{Theoretical dose calculations in microbeam radiation therapy}

The first Monte Carlo calculations in MRT go back to 1992, when Dan Slatkin [16] calculated dose distributions produced inside a human-head phantom. The use of "cylindrical" (circular) beams was initially very attractive, since much higher values in PVDR (Peak to Valley Dose Ratios) could be achieved. Most preclinical research during the last 25 years has been performed with microplanar beams [17-19], due to the ease of manufacturing collimators which produce planar beams. Early MC calculations benefited from advanced physics models [20]. E. A. Siegbahn [21] compared several MC codes, including PENELOPE, GEANT and the improved EGSnrc version, which were determined to be adequate codes for dosimetric studies in MRT due to their advanced low energy electron and photon tracking libraries. The issue of polarization was first studied by Felici and Hugtenburg [22,23]. I. Rovira-Martinez used a more recent version of PENELOPE including the polarization and incorporated a phase space file (PSF) from the specific geometry at the ESRF [24]. A final and comprehensive analysis of all important parameters like PSF, polarization and residual leakage radiation from the tungsten carbide MSC was published by Bartzsch et al.[25]. A possible improvement to the MC calculations might be the inclusion of the totally reflected photons interacting at grazing angles with the inner surface of the tungsten carbide MSC, which may lead to a small dose contribution of photons from that surface into the valley area. Their contribution can be estimated to be lower than $5 \%$ of the calculated valley dose. The most important progress and mandatory step to move forward with the proposed veterinary trials was the development of a fast TPS. A convolution based algorithm was introduced by Bartzsch [26] and implemented in the VIRTOUS platform [87]. In addition, the platform also allows a full MC calculation with microbeams of different sizes and center-tocenter (ctc) distances inside a phantom or a patient with existing CT data from a commercial unit, previously calibrated for the correct conversion in Hounsfield Units (HU).

The peak-to-valley dose ratio (PVDR) is a relative value, and consequently becomes important only when dose values are converted from the treatment plan to compute the absolute valley dose for the normal tissue, which corresponds to the classical maximum admissible dose value with respect to normal tissue complications. The strong influence of larger field sizes and tighter ctc spacing rapidly leads to very small PVDRs as shown in Fig. 1. Unpublished data by Laissue et al. indicate that for microbeam sizes between 25 microns and 75 microns FWHM, the adverse effects or normal tissue complications do only correlate with the valley dose and not with the peak dose. On the other side, preclinical studies did show, that a narrow microbeam ctc spacing is more effective for tumor growth suppression than a wide microbeam $[27,28]$. Comparable geometries were used; e.g. 50 micron width and 200 ctc versus 500 microns width and 2000 microns ctc, in order to keep the ratio of the direct cell killing from high peak doses in the unit volume constant. In this context it should be pointed out that several of the

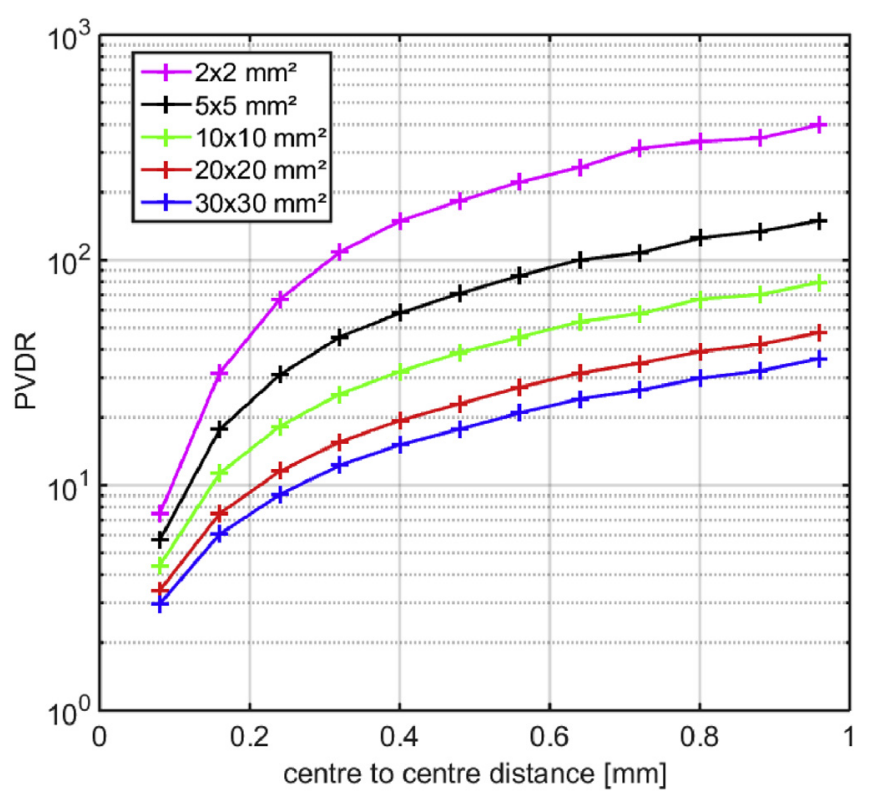

Figure 1. PVDR for different field sizes and center-to-center spacings.

MRT-specific effects are related to the surface area between high and low dose regions and the contact surface is certainly instrumental for the repair of heavily irradiated tissues in the peak regions.

From a theoretical dose calculation point of view, a compromise has to be found to solve the following problem: most preclinical studies could use small field sizes with a tight ctc spacing and high peak entrance dose values to achieve a superior tumor control probability (TCP), while the use of larger field sizes and tumor locations at greater depth using relatively low energy photons would oblige us to reduce the peak entrance dose values in ranges where a) the crucial contribution of the valley dose at the tumor is minimized and b) the differential effect on the tumor vasculature from the peak doses for several hundreds of Gy is reduced as well [29]. One possible option to overcome this problem may be to interspearse these microbeams from multiple ports, where larger ctc spacing for the normal tissue assures a sufficiently low valley dose and the tighter ctc spacing an optimized TCP [30] in the overlap region.

A comprehensive MC study comparing different field sizes, target sizes and geometries was performed by Anderson [31]. In this study a new definition in addition to the PVDR was introduced: PMVDR, the ratio between the mean doses in the peak region and in the valley region, which may be of interest when absolute valley dose values shall be respected in comparison to broad beam irradiations and may perhaps better represent the dose-volume relationship that MRT relies on.

\section{Experimental dosimetry}

The ESRF is perhaps the most suitable source for future clinical trials of brain tumors where the spreading of the microbeams due to cardiosynchronous movement of the tissues must be avoided by extremely rapid dose delivery. The only other place with concrete plans for clinical trials in MRT are at the Imaging and Medical beamline (IMBL) at the Australian Synchrotron in Melbourne. The very high dose rate at the ESRF represents a challenge in measuring the dose under broad beam configuration with an ionization chamber based on the recommendations in the International Atomic Energy Agency's TRS398 protocol [32]. The current protocol 
at the ESRF, which stays as close as possible to these recommendations, performs measurements in a PTW water tank at $2 \mathrm{~cm}$ depth for a $2 \mathrm{~cm} \times 2 \mathrm{~cm}$ field size using a PTW pinpoint ionization chamber with a volume of $0.015 \mathrm{~cm} 3$ (see parameters below) Table 1.

A standard cylindrical ion chamber cannot be used for synchrotron measurements owing to excessive ion recombination corrections, corresponding to more than $30 \%$ at such dose rates. Additionally, the source dimensions with a fixed, vertical beam height of usually 520 micron obliges us to scan the target as well as any dosimeter to be irradiated through the beam at a typical speed of $20 \mathrm{~mm} / \mathrm{s}$. The standard irradiation procedures for preclinical and clinical irradiations uses a GUI (Graphical User Interface) that automatically calculates (see Equation (1)) the correct speed for the microbeam irradiation as a function of the desired peak entrance dose to be delivered, the measured dose rate, the current in the machine, the slit size used and the selected micorbeam size, where output factors are tabulated from a Monte Carlo calculated library and scaled to the broad beam field conditions.

$v_{z(\mathrm{~mm} / \mathrm{sec})}=\dot{D}_{(\mathrm{Gy} / \mathrm{s} / \mathrm{mA})} \cdot I_{(\mathrm{mA})} \cdot O F_{(\text {output factor })} \cdot \frac{z_{\text {beam }- \text { height }(\mathrm{mm})}}{D_{(\mathrm{Gy})}}$

$v_{z(\mathrm{~mm} / \mathrm{sec})}$ : scanning speed.

$\dot{D}_{(\mathrm{Gy} / \mathrm{s} / \mathrm{mA})}$ : measured dose rate

$I_{(\mathrm{mA})}$ : machine current (storage ring)

OF: output factor scaling the peak entrance dose to the broad beam dose at $2 \mathrm{~cm}$ depth for a $2 \mathrm{~cm} \times 2 \mathrm{~cm}$ field size $z_{\text {beam-height }(\mathrm{mm})}$ : slit size used to define beam height.

$D_{(G y)}$ : desired peak entrance dose at $3 \mathrm{~mm}$ depth to be delivered.

The ion recombination correction using the two-voltage method assumes a constant full illumination of the detector, rendering this method unsuitable for our purpose. At the ESRF, we have devised a so-called ramping method reducing the current in the electron storage ring, which represents the only reliable way to reduce the dose rate at identical spectral conditions. This current ramping method allows us to determine the ion recombination correction resulting in values between $3.7 \%$ and $4.7 \%$ for an electron current in the synchrotron storage ring (SSR) ranging from 160 to $200 \mathrm{~mA}$. Similar results were found by independent measurements using Alanine dosimeters: $2.35 \%$ ion recombination correction at $160.7 \mathrm{~mA}$ and $5.5 \%$ ion recombination correction at $197 \mathrm{~mA}$ (see Fig. 2). The uncertainty in the absolute dose measurements using this methodology still exceeds the recommended 3\% for RT applications in humans. We foresee additional measurements using calorimetry to determine the dose rate at the ESRF to compare with our ion chambers.

The production of very regular microbeams is a crucial aspect for MRT to correctly predict the dose from CT data input assuming perfectly parallel beams all with an FWHM equal to 50 micron. After several variable MSCs (Archer collimator [17]), Tecomet MSC [33] the advanced ESRF MSC (EMSC) produced from a solid tungsten carbide piece using new wire cutting techniques [34] produces

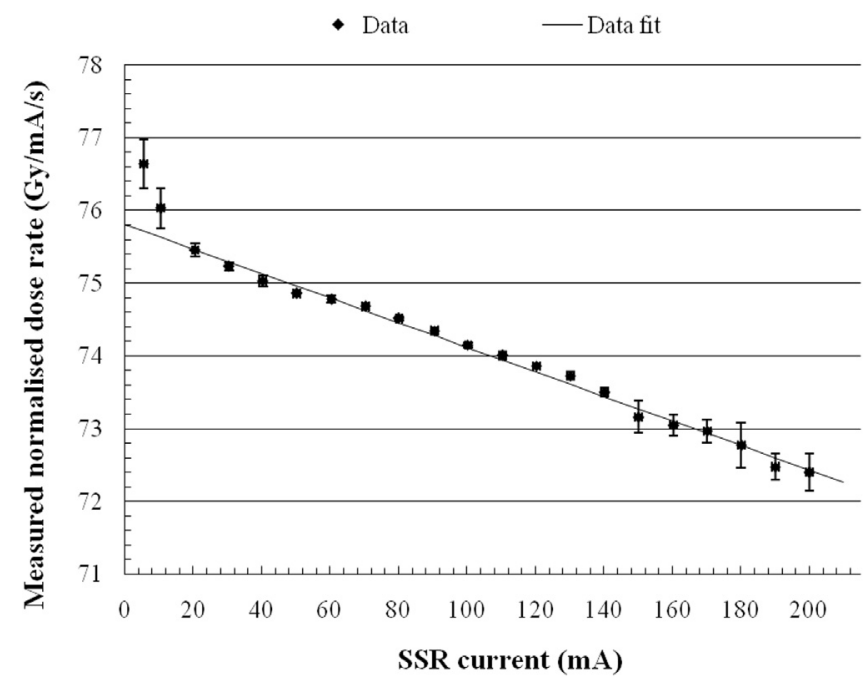

Figure 2. Dose rate measurements (normalised by the Storage Ring SR current (Gy) $\mathrm{mA} / \mathrm{s}$ )) obtained for different currents in order to determine the ion recombination correction factor.

perfectly regular beams of 50 micron with a deviation of \pm 1 micron. This was an important step to be able to assume identical sizes in FWHM of these microbeams as input data into the MC calculations for realistic dose computation.

The relative-dose profiles at depth can be determined using several types of detectors, all with their individual advantages and disadvantages [34]. The most promising results so far were obtained using Gafchromic films either in combination with a microdensitometer [35] or a modified Zeiss Axio Vert.A1 microscope.

Other potential high resolution dosimeters which are ideal candidates for the MRT dosimetry include fluorescence nuclear track detectors (FNTDs) from Landauer (Al2O3 detectors) demonstrating an excellent resolution [36,37], and a two-dimensional thermoluminescence (TL) dosimetry system consisting of LiF:Mg,$\mathrm{Cu}, \mathrm{P}(\mathrm{MCP}-\mathrm{N})$-based TL foils and a TLD reader equipped with a CCD camera and the large size planchete heater developed at the Institute of Nuclear Physics in Poland [38]. A large body of research and development over the last 15 years has gone into the development of Silicon-strip detectors [39-42] with the potential to develop an online monitoring system to simultaneously monitor the peak and valley dose during patient treatment. Such technical solutions are currently under further development in the frame of a collaboration grant with SINTEF and the Univ. of Wollongong (see section III e).

Further examples of new approaches to develop dosimetry systems to read high doses with high resolution include the use of samarium doped glasses from research groups in collaboration with the Canadian Synchrotron Radiation Facility [43-45].

For the first time the radiation induced optical absorption in five commercially available UV optical fibers under synchrotron

Table 1

Parameters used for the reference dosimetry protocol.

\begin{tabular}{ll}
\hline Parameters of interest for reference dosimetry & Values/characteristics \\
\hline Phantom material & Water \\
Ionisation chamber type & Cylindrical \\
Measurement depth $z_{\text {ref }}$ & $2 \mathrm{~g} \mathrm{~cm}^{-2}$ \\
Reference point of chamber & Central axis, at the centre of the cavity volume \\
Field size & $2 \mathrm{~cm} \times 2 \mathrm{~cm}$ \\
Scan speed & $20 \mathrm{~mm} / \mathrm{s}$ \\
\hline
\end{tabular}


irradiation was investigated as part of the COST Action "TD1205 "Innovative Methods in Radiotherapy and Radiosurgery using Synchrotron Radiation (SYRA3)", in order to evaluate the color center generation/recovery for use in radiation dosimetry. The irradiations were done at the ESRF synchrotron accelerator, in Grenoble, while the tests were carried out at the National Institute for Laser, Plasma and Radiation Physics, in Bucharest, Romania. The dose rates were $70.69 \mathrm{~Gy} / \mathrm{sec} / \mathrm{mA}$ and $65.49 \mathrm{~Gy} / \mathrm{sec} / \mathrm{mA}$ since the samples were exposed in two separate experiments. The total doses were varied between $5 \mathrm{~Gy}$ and $2000 \mathrm{~Gy}$. Under these conditions, three of the optical fibers proved to be radiation hardened, while two of them were sensitive to synchrotron radiation exposure. All the optical fibers showed a recovery of the optical absorption after storage for 10 days at room temperature [46].

A special setup was developed to monitor the dynamics of the color centers in the UV spectral range (Fig. 3). The samples sensitive to irradiation showed a linear dependence of the optical absorption at $\lambda=229 \mathrm{~nm}, \lambda=248 \mathrm{~nm}$, and $\lambda=265 \mathrm{~nm}$, for total doses between $60 \mathrm{~Gy}$ and $2000 \mathrm{~Gy}$, after the second exposure to synchrotron radiation. For both samples, the optical absorption remained almost unchanged at $\lambda=330 \mathrm{~nm}$ with increasing dose. By selecting in an appropriate manner the type of the optical fiber to be subjected to radiation and the dose rate, optical fiber based dosimeters can be developed for on-line dosimetry. As a novelty, the investigations included some $\mathrm{THz}$ spectral measurements of the irradiated samples, tests which highlighted the irradiation induced changes in the reflectivity of optical fiber samples.

Three selected detector systems are presented more in detail in this paper and plans within the current COST action SYRA3 include a study to compare the most promising MRT detector systems as well as advances in broad beam measurements. Sections III c, III d, and III e will give examples of recent developments and results in the field of high resolution dosimetry.

\section{Radiochromic film dosimetry}

Introduction: Radiochromic (RC) films are self-developing coloration detectors consisting of a radiation sensitive single or double layer of diacetylene microcrystals on a thin organic base. The diacetylene monomers join up upon irradiation, creating long polymeric chains responsible for the strong optical absorption $[47,48]$. The colorless active monomers display main absorption peaks at about $617 \mathrm{~nm}$ and $670 \mathrm{~nm}$ at room temperature) and its lithium salt (LiPCD) in EBT films (main absorption peak at $\sim 583 \mathrm{~nm}$ and $635 \mathrm{~nm}$ ). Various matters concerning radiochromic film dosimetry have already been reviewed in depth, notably published by the American Association of Physicists in Medicine [49-51]. However, taking into account the recent advances in the field, other topics of prime importance for the use of radiochromic films for therapeutic applications at synchrotron facilities still need to be investigated.

Spatial resolution and film readers: Dose assessment is traditionally based on linear absorbance measurements using often "white" light sources, such as those used in flat-bed colorscanners. The transmission (or reflection) image is analyzed for dose assessment in three wide color channels (RGB analysis) and the data obtained in either one of them (usually in the red one) or in all channels [52-54]. Alternatively, spot spectrophotometers, densitometers, and microdensitometers with light sources of appreciable spectral content in the region of intense light absorption are often used. Low power lasers, such as $\mathrm{He} / \mathrm{Ne}(632.8 \mathrm{~nm})$ and diode (e.g. 650-670 nm) lasers and broadband red-light emitting LED sources coupled with band-pass filters are often used along with either a photodiode or a photomultiplier.

Flat-bed scanners equipped with "white" light sources and arrays of charged coupled devices (CCD) are useful in the study of synchrotron radiation fields for various applications such as alignment procedures, uniform film irradiations and dosemapping. However, the currently available commercial flat-bed scanners have a charge-coupled device (CCD) with the required resolution, however the Modulation Transfer Function (MTF) of the system is not adequate to provide spatial resolution for dose profile measurements in MRT. In that case either spot microdensitometers or systems coupled with an optical microscope can be used.

Environmental effects: Molecular motions influence the structure of the polymer. Therefore, the shape of the absorption spectrum is influenced by the temperatures during film irradiation, storage and reading, usually shifting towards lower wavelength with increasing reading temperature.

Humidity and UV exposure (even by sunlight or light from fluorescent lamps) may also influence the film response by a degree which depends on the coating used among other things. Radiochromic films undergo post-exposure signal intensification, with the polymerization-rate decreasing with time. Adequate time has to elapse between irradiation and measurement to achieve accurate measurements. Thus sticking to a fixed carefully designed protocol is crucial to obtain reproducible and accurate dosimetric results with Radiochromic films.

Energy response: Taking into account that a) most of the imparted energy during the therapeutic uses of synchrotron radiation is related to photons of energy less than about $150 \mathrm{keV}$, and b)

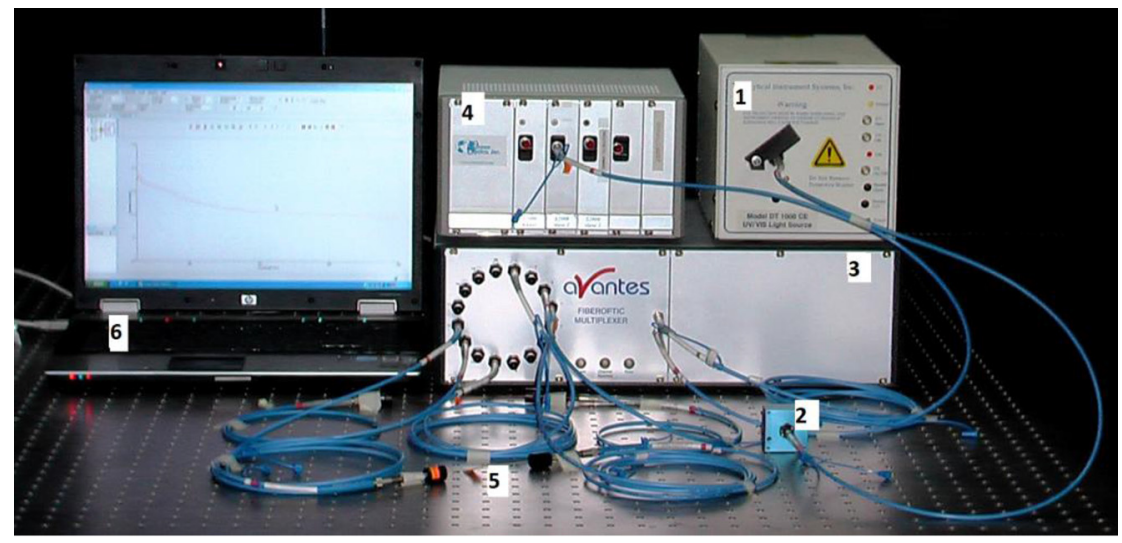

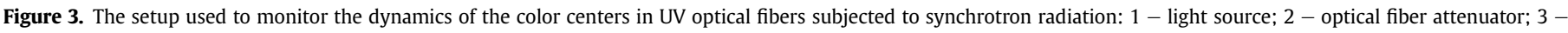
optical fiber multiplexer; 4 - optical fiber mini spectrometer; 5 - optical fiber sample; 6 - laptop. 
the photon spectrum varies with depth as well as between the peak and the valley region [55], some corrections may be necessary to cope with the energy dependence. To partially overcome the energy response of the films, calibration films are irradiated under identical conditions to those previously described in the dosimetry protocol, referring to absolute dose measurements.

Measurements carried out by Bartzsch [56] indicated a $50 \%$ decrease in the response of HD-810 films in water with decreasing photon energy from $100 \mathrm{keV}$ to $40 \mathrm{keV}$ and an about $15 \%$ increase in the response of HDV2 films in the same energy region. Similarly, simulations by Hermida-Lopez et al. [57] indicated that the EBT and EBT2 films exhibit an energy-dependent response in water in the energy region from 10 to $100 \mathrm{keV}$, with a $10 \%$ and $40 \%$ maximum reduction at $40 \mathrm{keV}$, respectively. They predicted that the EBT3 films would have a constant response within $2.3 \%$ over the entire energy region. However, in practice, one cannot exclude the potential existence of intrinsic energy-dependence, a factor usually not taken into account when using radiation transport codes. Thus, the radiochromic film energy-response has to be assessed experimentally.

Muench et al. [58] showed that the response HD810 films to 60 $\mathrm{kVp}$ X-rays (28 keVeff) is lower by about 30\% than that to $4 \mathrm{MV}$ Xrays. Kron et al. [59] reported that MD-55 films underestimated the dose by a factor of two when irradiated with a monoenergetic $26 \mathrm{keV}$ synchrotron-generated X-ray beam. Nariyama et al. [60] studying the energy response of MD-55 and HD-810 films reported measurable dose up to 50 and $400 \mathrm{kGy}$, respectively, and an under-response relative to 60Co gamma rays to low energy photons. In HD-810 films an almost constant under-response by $20 \%$ was observed in the energy region $30-100 \mathrm{keV}$, relative to Co-60 gamma rays, and a gradual increase in MD55-2 film from about $5 \%$ to almost $40 \%$ as the energy decreases in this energy region. Similarly, Cheung et al. [61] studying MD55-2 and HS film observed a gradual decrease of response with decreasing energy from 100 to $30 \mathrm{keV}$ up to about $40 \%$ and a large over-response (up to a factor of five at $50 \mathrm{kVeff}$ ) in XR-T RC films.

Oves et al. [62] observed in LiPCD-loaded EBT films a 0.76 and 0.81 response to 75 and $125 \mathrm{kVp}$ X-rays relative to $6 \mathrm{M} \mathrm{X}$-rays. Brown et al. [63] reported responses of EBT, EBT2 and EBT3 films to $35 \mathrm{keV}$ synchrotron-produced monochromatic beams of $0.76,1.24$ and 0.98 relative to $4 \mathrm{MV}$ X-rays, respectively. Similarly, comparing the output factors of X-ray machines in the energy range from 50 to $125 \mathrm{kVp}$ measured by EBT3 and a parallel plate ionization chamber Gill and Hill [64] reported differences up to only $3.3 \%$ in $2.0 \mathrm{~cm}$ fields. The differences were consistent with the estimated total uncertainty. On the other hand, Villarreal-Barajas et al. [65] irradiating EBT3 films with $70-300 \mathrm{kVp}$ X-ray beams reported a gradual reduction of the response with decreasing energy from 0.94 at 168 keVeff down to 0.79 at 32 keVeff using the red channel of RGB images and even lower using the blue one (0.83 and 0.74 , respectively). Moreover, Massillon et al. [66] found a dosedependent reduction in the response of EBT3 films to $50 \mathrm{kVp} \mathrm{X-}$ rays (20 keVeff) up to $11 \%$ relative to $6 \mathrm{MV}$ X-rays. In conclusion, even for films such as EBT3 that are often referred as dosimeters with no energy-dependence, extra care has to be taken when synchrotron beams are used for therapeutic purposes.

Microbeam fields: A significant number of investigators have exposed films in MRT to study among other things, relative output factors, transversal dose-profiles and depth-dose distributions. The measured valley doses in such profiles have in general been $10 \%-$ $15 \%$ higher than those predicted by MC simulations.

At the Spring-8 synchrotron in Hyogo, Japan, Crosbie et al. [35] irradiated two types of radiochromic films that differed substantially in their dose response (HD-810 and EBT), using an array of $25 \mu \mathrm{m} / 200 \mu \mathrm{m}$ microbeams (mean energy $120 \mathrm{keV}$ ). Using a Joyce-
Loebl micro-densitometer as a film-reader, they found that the PVDR in a solid-water phantom reached its maximum value at the depth of $1 \mathrm{~mm}$, decreased with depth up to $10 \mathrm{~mm}$ and remained practically constant at larger depths.

Martinez-Rovira et al. [24] irradiated HD-810 films with $50 \mu \mathrm{m} /$ $400 \mu \mathrm{m}$ micro-beams at the ESRF with a photon spectrum ranging from 27 to $600 \mathrm{keV}$ (mean energy $100 \mathrm{keV}$ ) and read them using a microdensitometer similar to the one used by Crosbie et al. The comparison of the PVDR values measured at various depths following irradiation with fields of various sizes with those predicted by simulations generally resulted in a lower measured PVDR value, reflecting approximately a $10 \%$ higher valley dose.

Finally, Bartzsch [56] also irradiated HD810 and HDV2 films with MRT beams also at the ESRF and read them using an inverted optical microscope coupled with a CCD-camera with a nominal spatial resolution of $5 \mu \mathrm{m}$. Films were also irradiated homogeneously at $2.0 \mathrm{~cm}$ depth in a solid-water phantom for calibration purposes. The doses given to these films were assessed by ionization chamber measurements employing the IAEA TRS398 protocol with marginal modifications. Film dosimetry indicated that the peak and valley doses up to $6 \mathrm{~cm}$ depth in the phantom were similar to those predicted by simulations within the measurement uncertainty. However, comparing the signal of the two film types, it was found that the peak dose values of the HDV2 films were slightly higher than those of the HD810 film, with an opposite situation in the case of the valley dose, resulting higher PVDR values when the HDV2 films were used. Such differences were attributed by the author to potential differences in the energy response. The investigator also observed, as anticipated, higher valley doses at the centre of the radiation field than close to its edges, resulting in smaller PVDR values in the central region of the field. Finally, in an attempt to simulate a two-field MRT treatment, dose measurements were carried out in an anthropomorphic head-phantom at distances of at least $1 \mathrm{~cm}$ from the skull. The deviations between the measured peak and valley doses at four studied depths and the predicted ones by simulations were below $5 \%$ in the peak region and between $10 \%$ and $15 \%$ in the valley region.

\section{Potential applications of PRESAGE ${ }^{\circledR}$ dosimeters and optical CT}

MRT represents a challenging dosimetry problem that requires measurements with both high spatial resolution and high dynamic range. Satisfying results have been achieved with the various dosimetry systems described in the other sections of this article and each approach has its individual advantages and disadvantages. However, for the eventual adoption of MRT in the clinic, we must add to our list of requirements the ability to make measurements over a large field-of-view (FOV) and in three dimensions. High spatial resolution often comes at the price of limiting both the region of space sampled and the dimensionality of the information obtained. For example, single detectors have limited sensitive areas and must be translated through the region-of-interest, involving a series of separate irradiations, rather than necessarily mimicking a single patient treatment. While having precise peak and valley measurements is very important, there is also an increasing need for 3-D measurements of dose as MRT irradiation geometries become more complex. A further important consideration is the need for comprehensive end-to-end verification of the entire MRT treatment chain. A dosimeter is needed that can follow the entire "patient journey", with multiple repositioning steps, from the initial X-ray CT scan, through planning with the newly developed TPS (as discussed in Section Medical Physics aspects in SSRT) to the final treatment. This would provide quality assurance not just for the apparatus and the physics involved, but also the software, workflow and operator. 2-D film dosimetry satisfies some of these 
needs, however, accurate read-out with a microdensitometer is tricky, time-consuming and involves multiple readout steps if films are stacked in 3-D.

For all of these reasons, methods of 3-D dosimetry complementary to the other approaches described in this article have been under active development over recent years. In the field historically known as gel dosimetry, two readout modalities have emerged more generally as leading candidates for quantitative dose imaging: Magnetic Resonance Imaging (MRI) of both radiochromic Fricke gels [67,68], and polymer gels [69]; and optical computed tomography $(\mathrm{CT})[70,71]$. Whilst the MRI-based techniques have been used successfully for dosimetry of SSRT protocols at the ESRF [72], they have proved unsuccessful for MRT, both because the gels themselves are not sufficiently robust to very high dose rates and because the available spatial resolution is not high enough to characterise microbeams of order $50 \mu \mathrm{m}[73,74]$. During the remainder of this section, we will focus on recent developments in the alternative method of 3-D optical CT microscopy using the radiochromic plastic polymer known as PRESAGE ${ }^{\circledR}$.

\section{Materials and methods}

PRESAGE $^{\circledR}$ is a solid plastic chemical dosimeter based on clear polyurethane mixed with a leucomalachite green reporter dye and a number of organic and/or metallic initiators [75]. A radiochromic reaction is induced after exposure to ionising radiation, resulting in a local change in optical density of the plastic. Effectively, the PRESAGE $^{\circledR}$ acts as a "3D radiochromic film" and the response to radiation is highly linear with dose at the normally imaged wavelength of $633 \mathrm{~nm}$, compatible with both HeNe laser and lightemitting diode (LED) light sources (see Fig. 4a and 4b).

Whilst details of the time-dependence of the dose response of PRESAGE ${ }^{\circledR}$ are currently the subject of active research $[76,77]$, the response is sufficiently rapid for the dosimeter to have a place in an online dosimetry system for benchmarking the MRT system and even prior to patient irradiation as evidenced by the video clip associated with Fig. 5 (supplementary multimedia resource).

Advantages of PRESAGE ${ }^{\circledR}$ include excellent spatial resolution, high dynamic range [78], dose-rate independence and the ability to record the dose distribution in three dimensions, giving much more flexible and realistic dosimetry. To date the highest resolution measurements have been made via fluorescent microscopy with pixel sizes down to $78 \mathrm{~nm}$ [79]. The corresponding disadvantages relate primarily to the fact that PRESAGE ${ }^{\circledR}$ is a chemical dosimeter with a relatively complex composition. A number of the constituents, particularly the polyurethane base, are supplier-dependent, with batches whose properties do not remain constant over time.

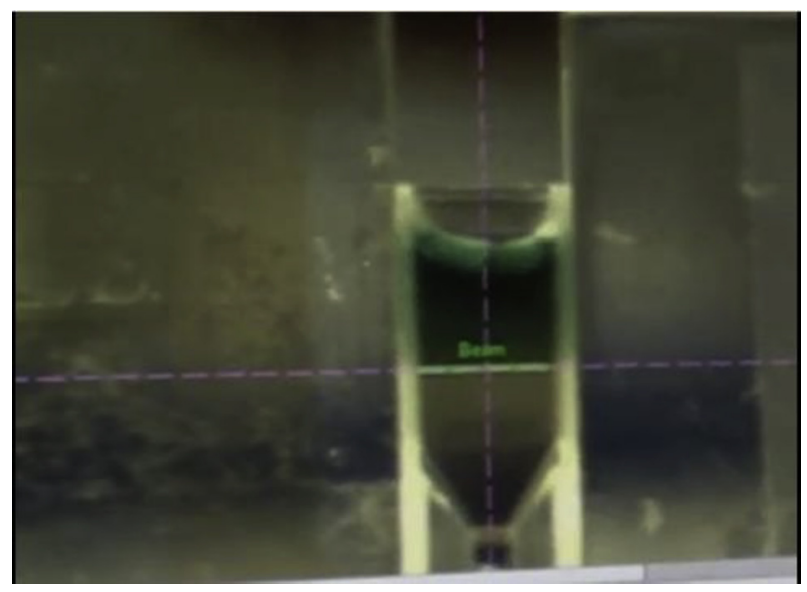

Figure 5. Still picture from real-time video taken on ESRF beamline ID-17, showing a sample of PRESAGE ${ }^{\mathbb{R}}$ changing colour in response to an MRT X-ray beam. Full video is available as a supplementary resource (https://www.dropbox.com/s/yxwin7sd6fmxetr/ Fig_PR2_movie.avi?dl=0, https://www.dropbox.com/s/d7kg8yxqb4nme85/Fig_PR4a_ movie.mov? $\mathrm{dl}=0$ and https://www.dropbox.com/s/aruuyayzhsyig2v/Fig_PR4b_movie. $\mathrm{mp} 4 ? \mathrm{dl}=0)$.

The manufacturer has also investigated a number of different formulations over the course of the research programme described here and the samples received have displayed differing sensitivities to radiation and ambient temperature, with variable degrees of time-evolution of their optical density post-irradiation. The interand intra-batch variability still needs to be investigated until the optimum formulation is found and characterized. Thus, whilst relative dosimetry is reliable [80], moving forwards from current results to absolute dosimetry will be challenging.

Development of the micro-imaging scanner has involved several upgrades during the programme to date. After an initial feasibility study [81], the system reported in Ref. [80] was able to reconstruct images of $512^{3}$ voxels from raw datasets consisting, typically, of around 1000 projections, each of $512 \times 512$ pixels, acquired in $1 \mathrm{~h}$ $10 \mathrm{~min}$. For the current system, this has been reduced to less than 3 min with the addition of a new camera (Zyla sCMOS, Andor Technology PLC, Belfast, UK) with a large pixel array and fast framerate. Reconstruction speed has been improved by the addition of an acquisition PC with 256 GB RAM, with the option of GPU acceleration. This time-frame for scanning makes it much more feasible to use optical CT as a beamline verification system for irradiations before treatment. The small size of the scanner and relatively low cost of the parts means it is possible to locate one inside the control hutch of the beamline. Other additions to the system are motorised a

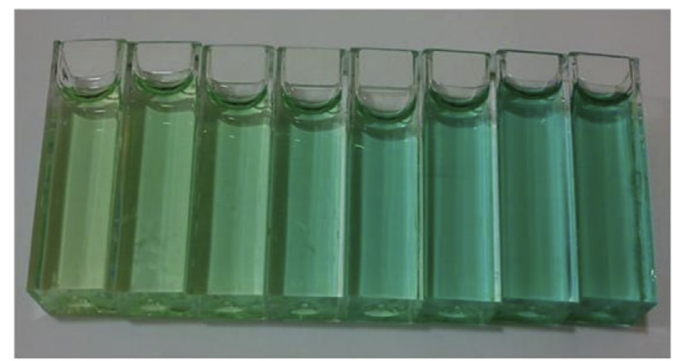

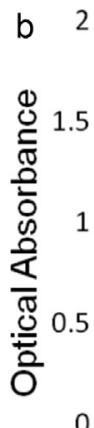

0

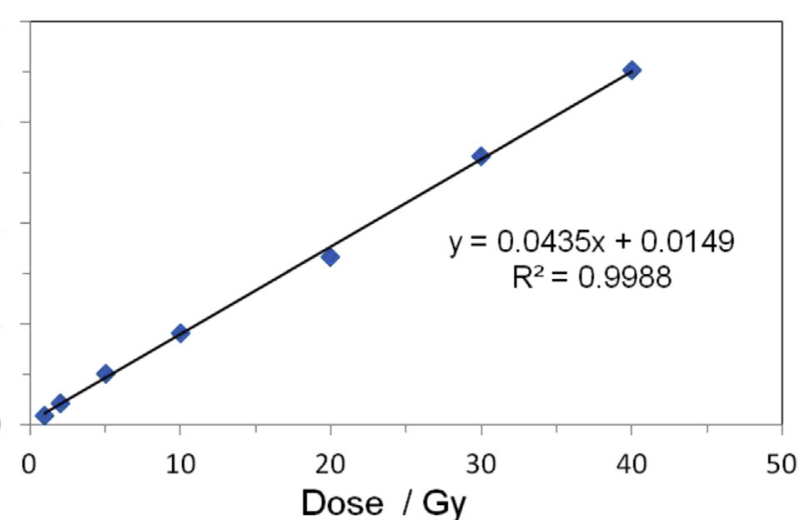

Figure 4. $\mathrm{a}$ and $\mathrm{b}$ Cuvettes of PRESAGE ${ }^{\mathrm{TM}}$ irradiated with a range of doses and the optical absorbance of the cuvettes as measured at 633 nm by a spectrophotometer. 
positioning stages and a sample mounting system, which allows reproducible positioning of individual samples. This potentially means absolute changes in optical density can be measured by registration of pre- and post-irradiation optical CT scans.

\section{3-D visualisation studies}

Irradiation of PRESAGE $®$ samples took place over two visits to the ID17 biomedical beamline at the ESRF and a general description of the irradiation conditions and protocols is given in Ref. [80]. The 3-D dosimetry programme has considered complex irradiations such as multi-port cross-firing [30], interlacing [82] and different collimation options, which are difficult to verify with planar dosimeters. Cylinders of PRESAGE ${ }^{\circledR}$ with diameters of $22 \mathrm{~mm}$ and $9.7 \mathrm{~mm}$, supplied by Heuris Pharma (Skillman, NJ) were irradiated using a variety of MRT geometries. Figs. $6-8$ show an example in which one of the $22 \mathrm{~mm}$ PRESAGE ${ }^{\mathbb{B}}$ cylinders was mounted inside a radiosurgery head phantom (Model 605, Computerized Imaging Reference Systems, Incorporated (CIRS), Norfolk, Virginia, USA). A

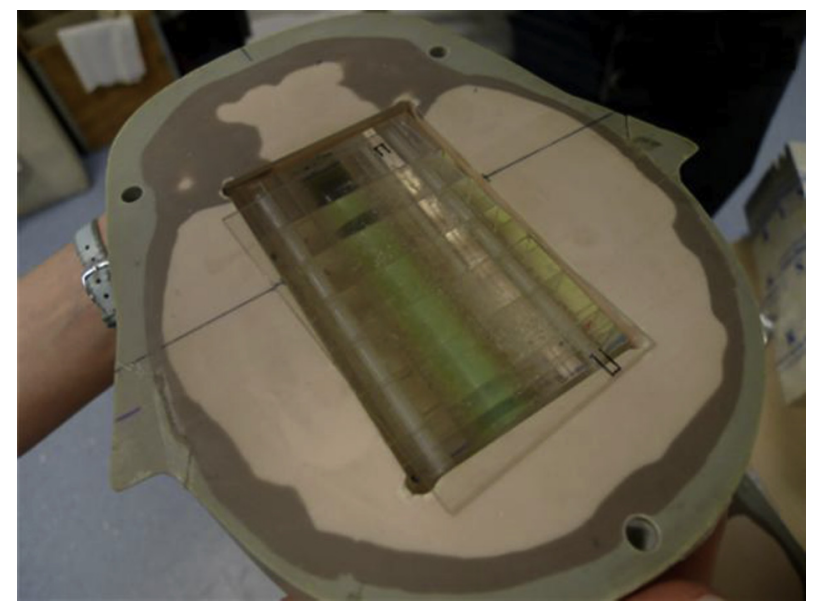

Figure 6. Example of PRESAGE ${ }^{\circledR}$ in use at the ESRF inside an anthropomorphic head phantom. The custom holder is divided into multiple plates, between which elements of a radiochromic film stack can be inserted, thus allowing independent measurements in 3-D for correlation between methods. Using the head phantom, it is possible to test the entire treatment process as described in the main text. cross-firing MRT treatment with three ports separated by $60^{\circ}$ angles was applied to the head phantom, with careful alignment of the phantom by eye such that the beams would cross in the centre of the PRESAGE ${ }^{\circledR}$ sample. The procedure simulated an attempt to hit a deep-seated tumor.

Data were acquired using the recently upgraded optical CT microscope. 1000 projection images of matrix size $512 \times 512$ pixels were reconstructed as a $512^{3}$ voxel volume with isotropic voxel size $20.8 \mu \mathrm{m}$. Fig. 6 shows the dosimeter in situ inside the head phantom, while Figs. 7 and 8 present the acquired 3-D data in a variety of formats. Each is useful for visualising different aspects of the dose distribution. The movies associated with Fig. 7 (available as online supplementary resources) provide a graphic illustration of the quantity of data acquired and the wide field of view covered, and they make it possible to visualise in 3-D the planar nature of the individual microbeams. By contrast, the multiplanar reformatting (MPR) of the data in Fig. 8 illustrates precisely why access to the full 3 -D data is so vital. The top row of images in Fig. 8 shows single planes through the dataset in, respectively, sagittal, axial and coronal orientations, which mimic the results one might expect to see from 2-D films positioned within the phantom in these orientations. Although the coronal image is easy to interpret in terms of the applied multiport irradiation, the sagittal and transverse images are more confusing. With a realignment of the imaging axes by just a few degrees - an operation that is simple but that needs to be very precise and would be virtually impossible with physical 2-D films, given the extremely narrow beams - the interpretation becomes straightforward. Considering the bottom left image ("sagittal-oblique"), we see that there are three grey levels: the lowest level corresponds to a single microplanar beam and is visualised as a solid rectangle from end to end of the sample and occupying the entire diameter. The mid grey corresponds to a set of lines on which exactly two microplanar beams cross, whilst the bright lines in the middle are the loci of points where all three microplanar beams cross, leading to three times the radiation dose.

The bright white "flecks" in Fig. 7 correspond to microscopic imperfections in the PRESAGE ${ }^{\circledR}$ samples. These lead to high absorption and there is a need for ongoing research to create improved samples. Similarly, the black streaks below the dose distribution in the left hand panel illustrate image artefacts arising from the back-projection reconstruction, which also need addressing.
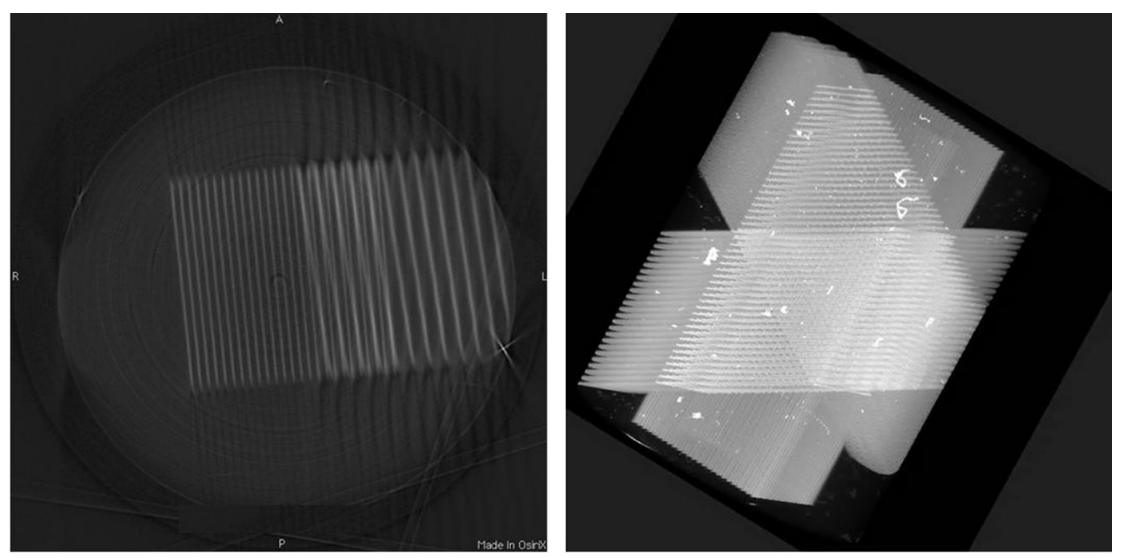

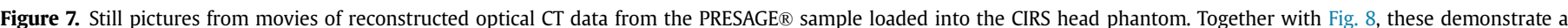

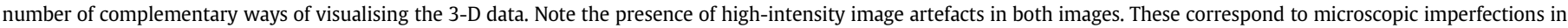

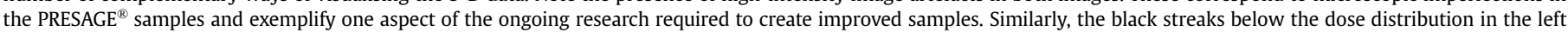

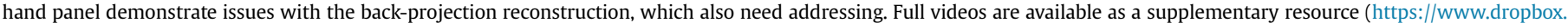

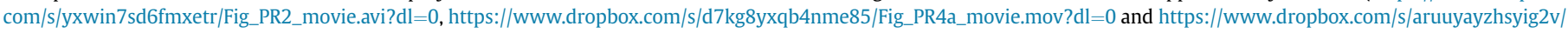
Fig_PR4b_movie.mp4?dl=0). 

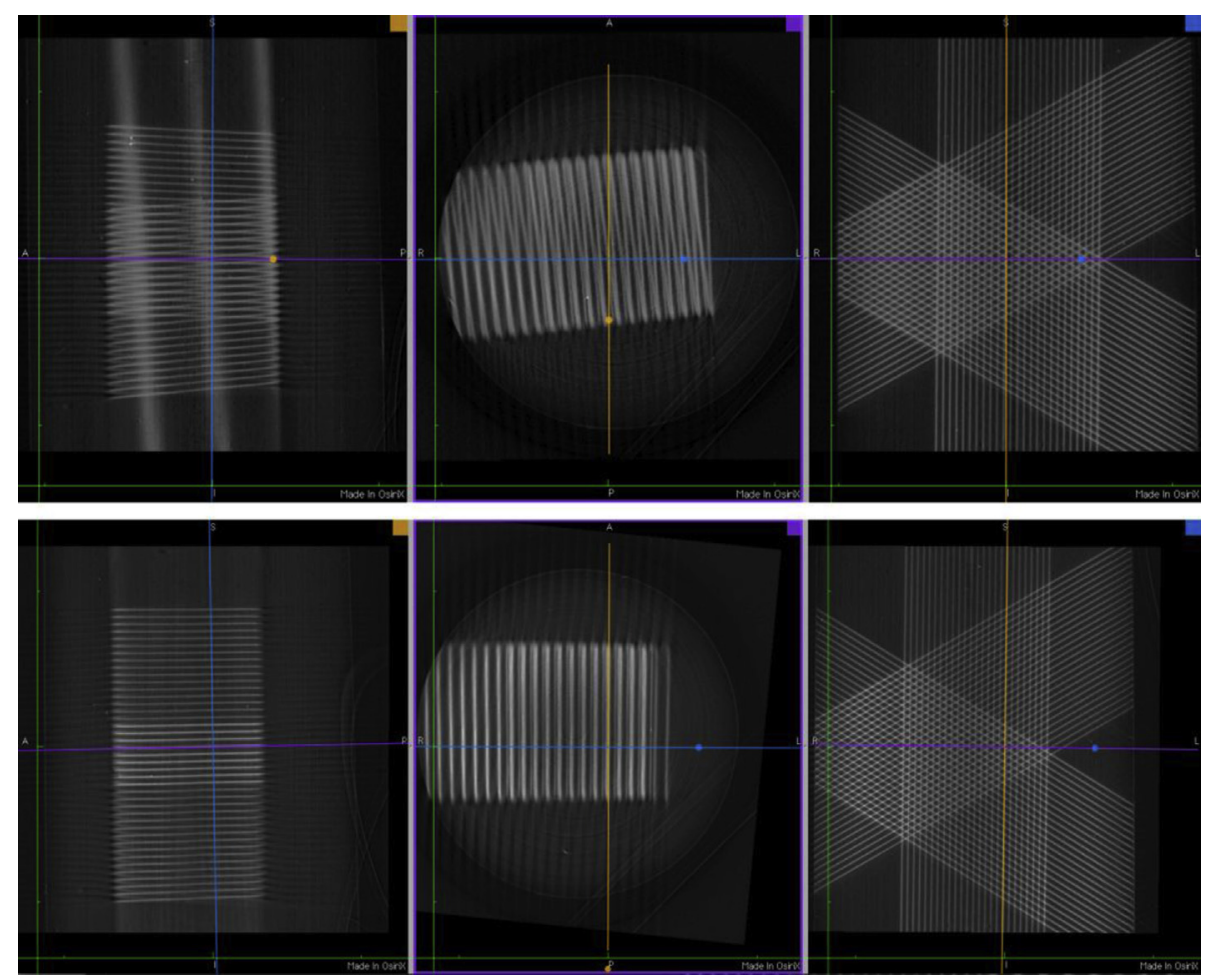

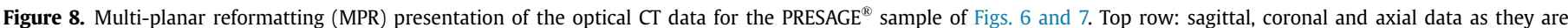

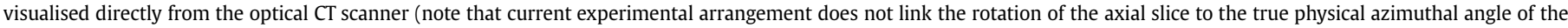
sample during irradiation). Bottom row: same dataset after minor axis tilts.

Despite these minor inconveniences, the data are of high quality and it is straightforward to verify whether radiation has been delivered to the required location in sample. Notice that the two angled beams are asymmetrically distributed in the images, such that the region irradiated by all three beams is triangular instead of hexagonal as intended. This represents an offset of approximately $2 \mathrm{~mm}$ in the crossing point of the beams. Such deviations from plan are extremely hard to deduce during the course of the experiment from films placed on the proximal and distal surfaces of the phantom during irradiation and this study emphasises the difficulties of performing such treatments on nonsuperficial tissues.

One valid and immediately available function of optical CT is therefore simply to act as a non-quantitative adjunct to other more accurate forms of dosimetry. In this mode, optical CT is already more than capable of simple "hit or miss" assessments, as well as quality assurance of other aspects of the delivery, such as the microbeam width and spacing, together with appropriate synchronisation of the shutter opening and goniometer motion.

At the time of writing, an automated patient positioning system has been developed, but is not yet implemented in the MRT Graphical User Interface (GUI) at the ESRF [83]. However, a treatment planning system is now in place and a clear goal for the future use of PRESAGE $\otimes$ is in contributing to the commissioning of any fully integrated planning and positioning system for conformal image-guided MRT from several ports, as described in Section Theoretical dose calculations in microbeam radiation therapy and section Towards conformal image guided MRT.

\section{Quantification and resolution issues}

The role outlined above, while important, is unnecessarily limited. As has been shown previously [84], optical CT is also a fully quantitative modality, with a linear response over a dose range of at least 10-80 Gy. Two issues remain to be resolved before optical CT can be used to verify quantitatively 3-D MRT treatment plans: (i) limited spatial resolution; and (ii) the methodology for aligning imaging and simulation data.

The resolution problem has been previously investigated [84] where it was found that the apparent dose-response of the optical CT system can vary with width of irradiated "slit" patterns. The measured peak dose is lower than expected and the valley doses correspondingly over-estimated due to blurring effects as the slit width decreases. This is a straightforward manifestation of the modulation transfer function of the imaging system - which can be measured in a variety of ways [80] - illustrating the fact that significant changes in pixel value occur even for structures that are several times the nominal spatial resolution. Whilst this type of effect is commonly tolerated in qualitative diagnostic imaging, resulting as it does in a reduction in image contrast, it leads to serious problems in the quantitative imaging of microbeam radiation dose.

Although the microbeams are easily visualised, early attempts to measure the PVDR gave significantly lower results than expected from Monte Carlo and film measurements for the reasons illustrated above. A beam profile measured using our original microscopy system is seen in Fig. 9a. The microbeams were nominally $50 \mu \mathrm{m}$ in width, with a center-to-center (ctc) distance of $400 \mu \mathrm{m}$ and field size $(3 \times 3) \mathrm{cm}^{2}$ deposited in a $9.7 \mathrm{~mm}$ diameter PRESAGE ${ }^{\circledR}$ sample. The ctc distance was measured by optical CT to be $(390 \pm 20) \mu \mathrm{m}$, in good agreement with the expected value. However, the PVDR estimated from this dataset at a depth of $143 \mathrm{~mm}$ was 4.2 with a range [3.3, 6.1]. By contrast, for a broadly similar depth of $100 \mathrm{~mm}$ in a $(3 \times 3) \mathrm{cm}^{2}$ field, the measured PVDRs were $15 \pm 3$ (Monte Carlo) and $13 \pm 2$ (film) [24]. For various technical reasons, the optical CT scans measured at that time had an uncertain baseline, as indicated, which 

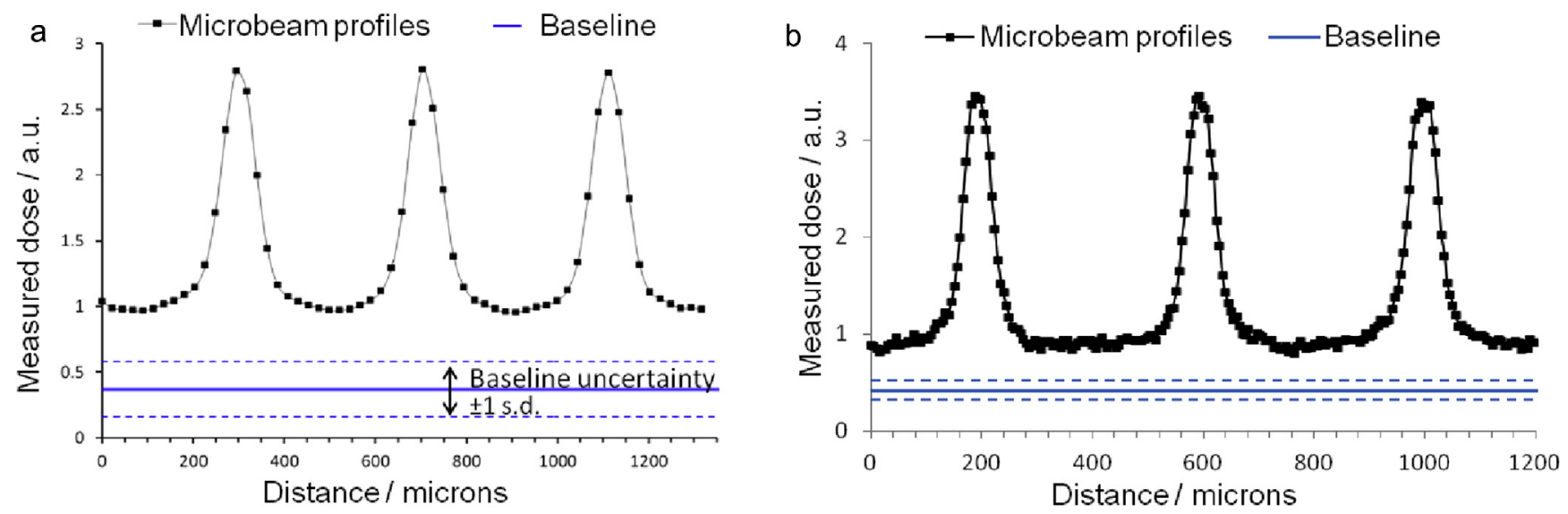

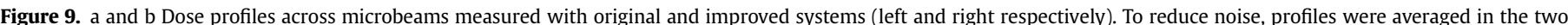

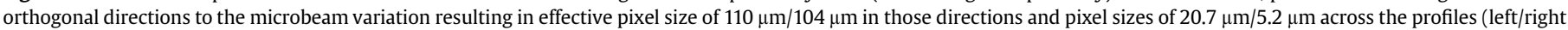

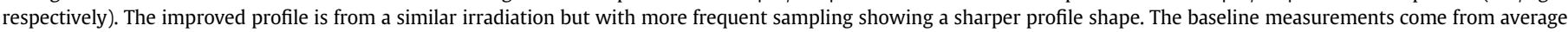
unirradiated regions on other samples, hence the uncertainty.

led to a measurement of PVDR whose large range was dominated by the consequent uncertainties in the valley dose. This can be resolved in the future if a system is available at the ESRF so an accurate prescan of the sample before irradiation can be acquired. The more serious issue is that the mean value is only $30 \%$ of what is expected, because of the effects of the limited spatial resolution. From a basic simulation of our system we have found that for beams of width $50 \mu \mathrm{m}$, a spatial resolution of $10 \mu \mathrm{m}$ or better is required to measure the true peak value after filtered backprojection reconstruction.

Given the fact that having a measurement of how the PVDR varies with depth would be very helpful, we have tried to improve the system to achieve better resolution and obtain more accurate peak and valley measurements. The experiment of Fig. 9b makes use of the full matrix size of the new camera (2048x2048 pixels, 3200 projections) and an optimal magnification, resulting in a reconstructed voxel size of $(5.2 \mu \mathrm{m})^{3}$. As the matrix size of the individual projections increases, more projections are required to satisfy the Nyquist sampling criterion, leading to an increase in scanning time to $30 \mathrm{~min}$ for this case. For lower noise data, five projections were averaged for each angle resulting in a final scan time of $1 \mathrm{~h}$. Profiles were measured at $40 \mathrm{~mm}$ depth (see Fig. 9b) giving a PVDR of $6.4 \pm 1.5$ (expected values $17 \pm 2$ Monte Carlo, $13 \pm 3$ film [24]). Although this new measurement seemingly represents only a small improvement and the true value of the PVDR is still an underestimate by more than a factor of 2 , the valley is noticeably flatter in the new results and the density of measurement points is now sufficiently high to capture the true peak, once the blurring effects introduced by the optical imaging chain have been removed. At the moment, the peak measured is still lower than expected due to the limited MTF of the optical system. The next step in the research is to make more accurate measurements of the imaging point-spread function and use these to deconvolve the raw data. Early indications (data not shown) suggest that by so doing, we will recover a value that more closely matches the data available from other techniques.

As alluded to earlier, the other unsolved question for PRESAGE ${ }^{\circledR}$ dosimetry is how to compare the results of 3-D imaging of this type with a 3-D MRT treatment plan. Clearly, the exceedingly small diameter of the microbeams means that alignment of datasets will be a hugely challenging problem. It remains to be determined what the appropriate quality assurance measures will be that correspond to the ubiquitous gamma analysis in routine external-beam radiotherapy [85]. Despite the improved resolution, absolute dose measurements of the peak dose are not yet feasible with our PRESAGE $®$ dosimetry system, but the determination of the valley dose remains the most important parameter, since it represents the threshold dose for the normal tissue tolerance. For such important benchmarking experiments, the PRESAGE ${ }^{\mathbb{R}}$ dosimetry system is currently the best choice for ultimate confirmation of a 3-D valley dose distribution prior approval of a treatment plan.

\section{Si-based multiple strip detector systems}

In order to meet the safety standards in treatment planning and quality assurance for preclinical MRT trials, an emergency beam shutter must be in place in case of any beam anomalies. The primary role of the beam monitor is to be one of several active and passive emergency interruption mechanisms designed to respond instantaneously upon detection of any abnormality in the MRT beam delivery. The beam monitor is coupled directly to readout electronics with a rapid time response, so as to be a real time online monitoring system. Silicon radiation detectors, manufactured in well-established technologies have been widely used in X-ray detection for over 20 years. Current available technology allows the feasibility of direct coupling of silicon sensors to their associated readout electronics with extremely high spatial resolution; thus providing an attractive solution as an active beam monitor for MRT.

\section{Multiple-strip detector for MRT}

The PVDR is a very important quantity to be monitored during an MRT treatment. Within the framework of the 3DMiMic project [86], a novel silicon sensor with multiple strips (or channels) has been proposed to monitor the X-ray beams that make up an entire microbeam array in MRT. Due to the extremely high dose rate in MRT, one key issue when using conventional silicon sensors is the large amount of charge generated by the X-ray photons. Upon exposure to an array of microbeams, the unusually high level of generated charge in a conventional silicon sensor will saturate the entire readout system. Moreover, the generated charges then diffuse in all directions, resulting in an inability to distinguish both the position and the intensity of the X-ray microbeam. Silicon sensors with various detailed configurations have been designed to address these issues by taking four key strategies. 
a) A $10 \mu \mathrm{m}$ thick sensor with short strip length was designed to reduce the available ionization volume. Any beam perturbation as well as the heat load on the silicon sensor is expected to be minimized using this approach.

b) Isolation structures such as 'guard-ring' and 'p-stop' (a heavily doped region that provides high recombination probability of the generated charge) were designed to surround each individual strip to limit the amount of charge reaching the readout electronics, as shown in Fig. 10. In addition, the guard isolation reduces the charge sharing between adjacent strips for better identification of an X-ray peak intensity position measurement.

c) Low resistivity substrates with a high doping concentration were investigated. The high concentration of dopants increases the recombination rate of the generated charge upon ionization thus resulting in a lower total collected charge and generated signal.

d) Particular care was taken in assuring the longest possible lifetime of the devices. The expected radiation damage to a silicon detector for the X-ray photon energies involved in the MRT is mostly related to the charging up of the surface oxide layers. In order to assure a proper operation of the sensor after very large radiation doses, specifically tailored p-spray and p-stop implantations were used in order to assure interchannel isolation and maximise the operating voltage. A small amount of bulk damage will also be present but this will not cause any concern due to the very large magnitude of the signal generated by the MRT beam. Periodical recalibration of the sensor will take into account efficiency losses due to bulk damage without compromising the operation of the system.

Based on the design strategies listed together above, several sensor designs were implemented, and a set of silicon wafers with high doping concentration levels were fabricated at SINTEF MiNiLab. A wet chemical isotropic etch that is well-established in silicon fabrication technology was used, and is the most critical step so as to reduce the sensitive volume down to $10 \mu \mathrm{m}$ thick. Special attention was paid to the mechanical design of the sensor to maintain their mechanical stability and the feasibility for general handling. Prior to the fabrication, the proposed designs were fully investigated using device simulations, performed on a small region of the overall strip design as indicated in Fig. 10.

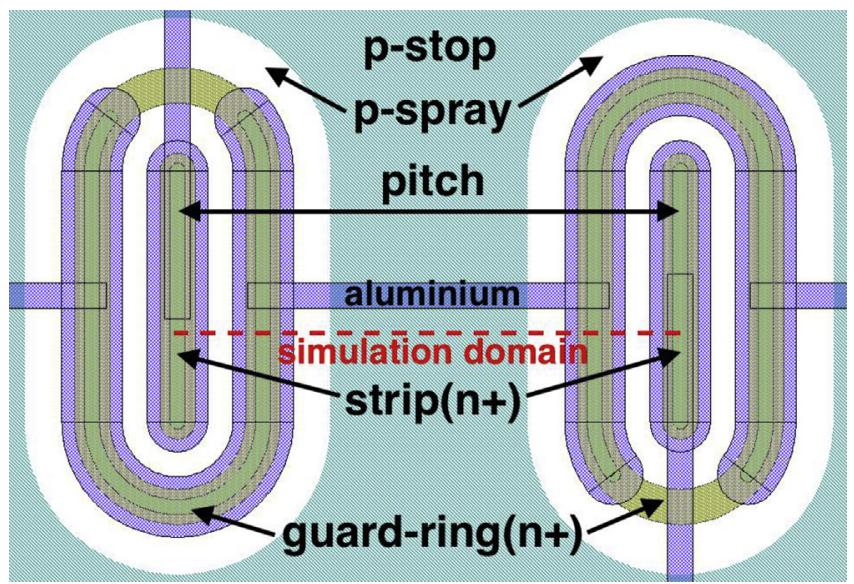

Figure 10. Design layout of one implemented strip design, showing the single strip that monitors the peak intensity that is surrounded by the isolation structures (guardrings and p-stop) to address the saturation issues due to the high dose rate in MRT.

\section{Sensor, assemblies and test set-up}

The fabricated devices were screened by parametric testing such as current and capacitance measurements. Working devices were then selected for experimental testing with microbeam arrays at the biomedical beam-line ID17 at the ESRF. Each sensor was mounted on a printed circuit board (PCB), compatible with the chosen 256-channel readout system that has the capability to read out all the channels on a 128 - or 256- channel sensor. The system is based on a commercially available analogue front end readout chip (AFE0064 from Texas Instrument), that was designed and configured by the Centre of Medical Radiation Physics, University of Wollongong, Australia. One of the wire-bonded detectors mounted on a PCB is shown in Fig. 11. Detailed studies of the sensors on device level are currently on-going through comparison with simulation results.

Six sensors of various designs were mounted and tested at the ID17 beamline over two periods, September and November 2014. The performed experiments aimed to identify the most suitable sensor configurations for MRT, and to provide a verification of the sensor functionalities and on-line capabilities. Fig. 12 shows the experimental setup at the beamline where the sensor assembly is

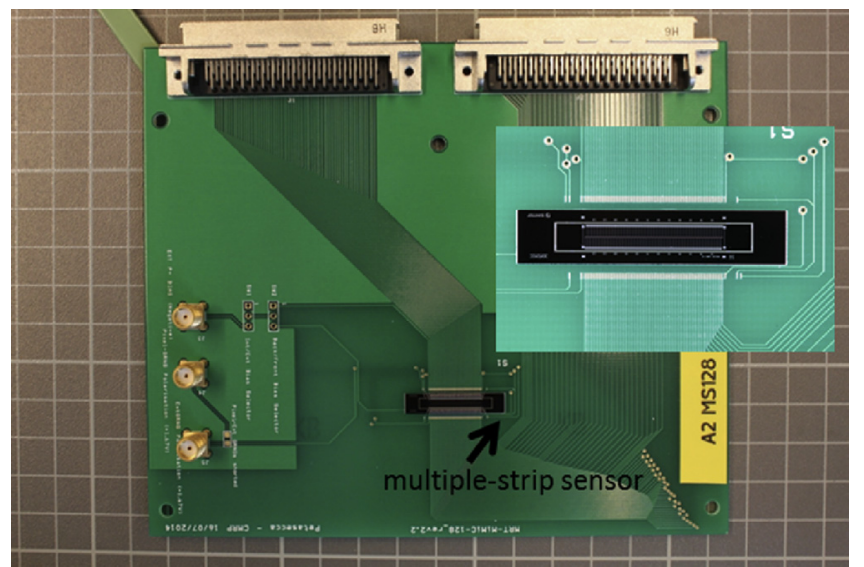

Figure 11. A sensor mounted on a printed circuit board compatible with a 256-channe readout system.

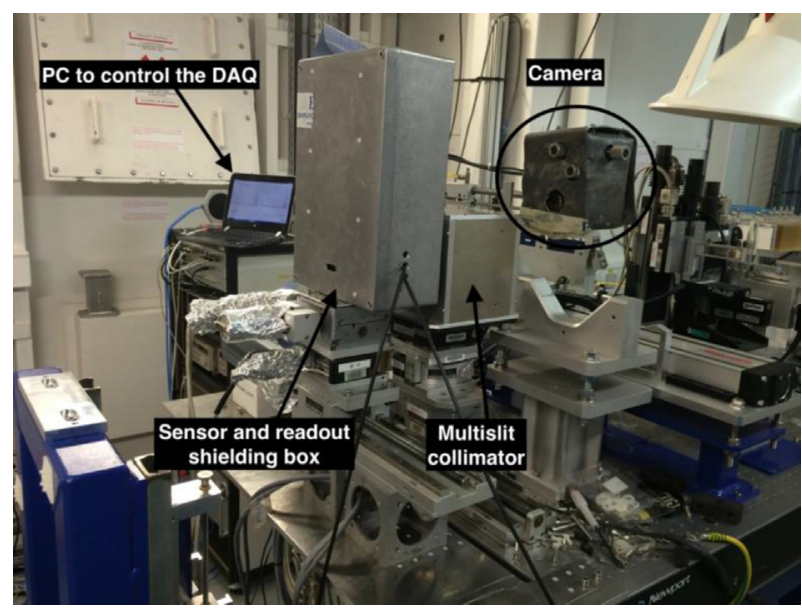

Figure 12. Experimental setup at ID17 at the ESRF. The sensor assembly is connected to the multichannel readout system and inserted in a shielding box mounted on a motorized stage, placed close to the multi-slit collimator that provides the microbeam array. 
placed in an aluminum shielding box in a fashion that can be connected to either a multi-channel readout or a single-channel electrometer. The entire assembly was then secured onto a motorized stage with $10 \mu \mathrm{m}$ precision in the $\mathrm{x}-, \mathrm{y}$ - and $\mathrm{z}$-directions, and was placed as close as physically possible to the multi-slit collimator (MSC) that striates the homogeneous synchrotron generated X-ray beam into an X-ray microbeam array in order to minimize the effects of beam divergence. The MSC to sensor distance was estimated to be between 15 and $20 \mathrm{~cm}$ in this experiment, giving a beam divergence of roughly about $1 \mu \mathrm{m}$ between adjacent microbeam peaks.

\section{First results from ID17 at the ESRF}

Two measurement approaches were used in the experiments that took place at the ID17:

1. Study of one single channel with respect to an array of microbeams by measuring the generated current in a single strip using an electrometer.

2. Study of the behaviour of a multiple strip sensor with respect to an array of microbeam using a multiple channel readout system that provides both the position and beam intensity of the entire array

\section{Single channel measurement}

In the single channel measurements, the generated current in one single strip and the isolated guard-ring structure were measured by two separate electrometers. The device was then scanned across an array of 25 microbeams, spaced by a nominal pitch of $400 \mu \mathrm{m}$ (as defined by the microbeam collimator). The generated current in the strip was recorded at a time interval that is sufficient for the device to shift by $10 \mu \mathrm{m}$ in the x-direction. One complete scan would therefore consist of 1000 current measurements that correspond to the $\mathrm{x}$-direction of the array at a step of $10 \mu \mathrm{m}$; a simple method that can demonstrate the capability of beam monitoring in a single strip without the complexity of a multi-channel readout system with the radiation induced current measurments acting as an independent calibration of the current measured by the multichannel readout system. Sensors with different designs such as strip length and different doping concentrations were tested using this approach. Fig. 13 shows one of the obtained measurements for a single strip operated in passive mode (no bias voltage was applied on the sensor) that has a strip length of $100 \mu \mathrm{m}$ and a resistivity of $5 \Omega \mathrm{cm}$ while the wiggler gap was set to be $40 \mathrm{~mm}$. The current recorded was interpreted as current counts, an arbitrary unit given by the data format on the instrument readout system at the ESRF. This first measured result clearly demonstrated that all 25 microbeams were correctly reconstructed by the single strip despite the scanning step limited by the motor precision (Fig. 13a); this is further illustrated by a more detailed plot of a single peak in Fig. 13b. Simulation studies of the charge collection efficiency profile (the response function) have been performed [86], and the charge collection profile is quite smeared, but with an FWHM (Full Width at Half Maximum) that is consistently $6.4 \mu \mathrm{m}$.

A simple model of the response can be made by assuming that the charge collection efficiency function is Gaussian around the strip, and that the microbeam intensity profile is 'box shaped' (uniform over $50 \mu \mathrm{m}$ ) with a sharp cut-off at the edges. The fit result gives a width of the microbeam of 49.5 microns, and a sigma of the gaussian of 9.3 microns, and is shown in Fig. 13b. The fitted values are very reasonable, given prior knowledge of the sensor and the microbeams. The disagreements observed, particularly in the valley regions are to be expected. The assumed sensor response function and the intensity profile are very simplified models. Further investigations are needed to fully understand the details, in particular in regions where the valleys are approaching the edges of the microbeams. It is also to be noted that the pitch of the microbeams at the detector location is found to be about $401 \mu \mathrm{m}$ consistent with the slight divergence of the synchrotron generated $\mathrm{x}$-ray beam.

Although the recorded current counts demonstrated the ability of beam profile reconstruction in a single strip, the actual current values are crucial in choosing the most suitable sensor configuration in terms of signal saturation in a final readout system. The radiation induced generated current in a single strip current was therefore recorded manually and was found to vary from a few nA to $100 \mathrm{nA}$ depending on the wiggler gap that was implemented and the synchrotron storage ring current. The generated current from the isolation guard ring structure were also recorded to be a few $\mu \mathrm{A}$ when the wiggler gap was set to be $24.8 \mathrm{~mm}$.

\section{Multiple channel readout}

The second measurement approach was performed using a 256channel readout system. The readout system integrates the input (generated current from the sensor), which is then sampled at an
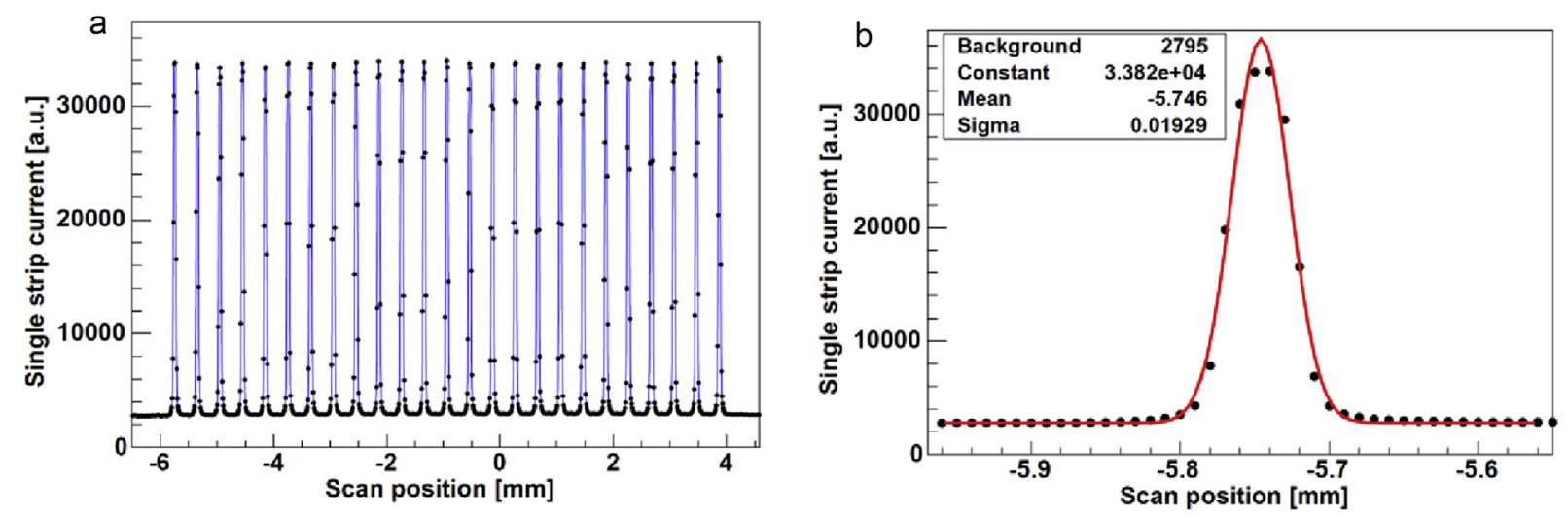

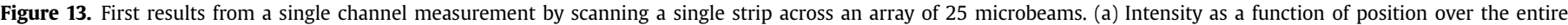

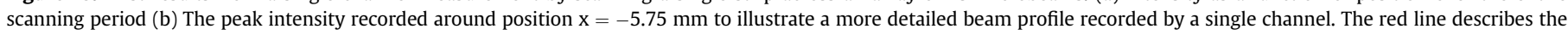

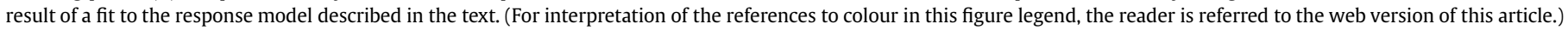


adjustable rate to provide various readout capabilities. The maximum charge that could be integrated per sample was $9.6 \mathrm{pC}$. Given the sampling rate, and charge collected per sample relative to the full range, the sensor current can be estimated. The key goal of this measurement is to investigate whether the designed multiple strip sensors can provide an accurate peak intensity measurement of an entire microbeam array; the ultimate goal of having a realtime on-line beam monitor for the MRT treatment. The measurements were performed with an array of 33 microbeams centered in the middle of the sensor (channel 64) where the sensor was exposed to the X-ray microbeam array for 2 s. Fig. 14 shows the mean current per channel recorded at an sampling rate of $1 \mathrm{kHz}$, for a wiggler gap of $24.8 \mathrm{~mm}$. In this case, the length of the sensor strips was $250 \mu \mathrm{m}$ (microbeam X-ray beam height 520 microns), and the material resistivity was $5 \Omega \mathrm{cm}$. The largest charge depositions per sampling of peak intensities were at around $17 \%$ of full range, while those in between to microbeams (typically referred to as the valley) were around $0.5 \%$. These signal levels were clearly offset from the 'no beam' samplings which were around $0.2 \pm 0.1 \%$. Further investigations (gain linearity, zero level offsets, crosstalk between strips etc.), are necessary before estimating reliable PVDRs.

The currents appeared to be rather uniform around the center of the array but gradually decreasing on the left- and right-hand side edges of the array. The roll-off in the measured peak current is primarily due to the small mismatch of the microbeam peak pitch (402 microns) and the silicon strip detector pitch (100 microns) at the measurement position. This mismatch is caused by the X-ray beam divergence. The mismatch increases to the point where the strips at the edges appear to be significantly misaligned with the microbeams. When a microstrip is aligned at the edge of a microbeam, only half the intensity will be seen, relative to the intensity seen in strip well aligned in a microbeam centre. With the detector and microslit designs at hand, this effect can only be avoided by placing the sensor as close as possible to the MSC. The latest test results have shown great improvements when the MSC to sensor distance is reduced by $10 \mathrm{~cm}-10 \mathrm{~cm}$. This confirms that the ideal solution will be to mount the sensor inside the MSC box within close proximity to the multislit collimator.

The collected data obtained for various sensor configurations as well as different operating conditions are currently being studied in details. The analyzed data together with a comprehensive study on sensor level will be published in a separate article. Further tests are also in the pipeline in the coming months for both full sensor assembly and simplified test devices that have only a few channels.

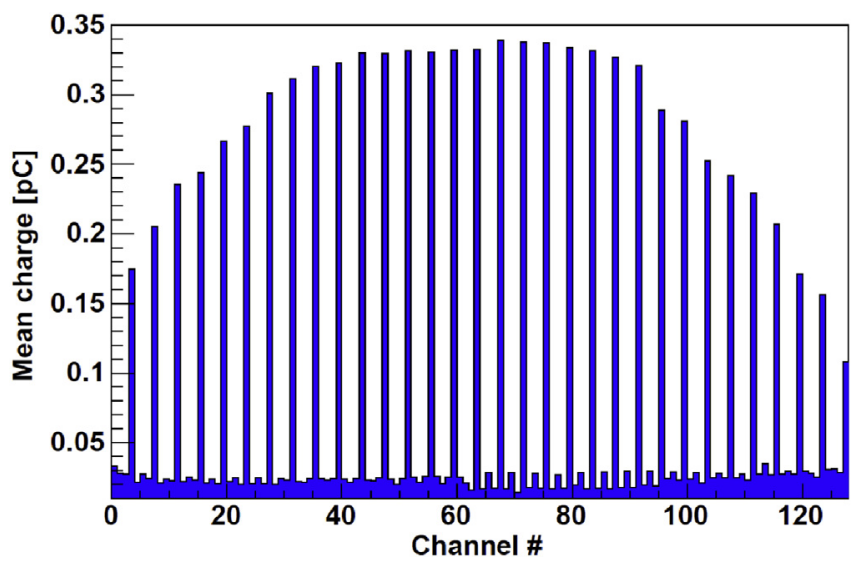

Figure 14. Average currents from 2000 samples over $2 \mathrm{~s}$ in each channel in a multistrip sensor with $250 \mu \mathrm{m}$ strip length. The uneven profile of responses is explained by cumulative misalignment between strips and microbeams.
Performance characteristics such as charge collection efficiency and post radiation damage performances will be carried out. The comprehensive test plan will identify the ideal silicon sensor configuration that can potentially be integrated into the ESRF control system, specifically for the MRT treatment.

\section{Towards conformal image guided MRT}

An image-guidance protocol has been developed [83] which allows for precise conformal microbeam irradiations from multiple ports, a technique that is mandatory for treating deep-seated tumors with MRT at ID17. By knowing precisely the tumor position inside the patient, the safety margins for the irradiation field may be reduced, thus increasing the PVDR and sparing especially the healthy tissue at the beam entrance. The same positive effects arise from the application of conformal radiation fields, which are adapted to the tumor outline, in contrast to the rectangular fields that have been applied in preclinical research so far.

The presented protocol is an extension of a protocol used at the Animal Oncology and Imaging Center in Hünenberg, Switzerland, and is based on the use of lead pellets attached to animal patients (typically cats and dogs) on prominent anatomical spots and on a bite block. These markers are in place during CT imaging for treatment planning and during the treatment session. The treatment planning platform VIRTUOS [87] is used for treatment planning, where the target volumes and the positions of the lead markers are identified and the directions of the treatment beams are defined.

Prior to irradiation, stereoscopic radiographs are taken of the patient on the sample stage, where the lead markers serve as clear reference points. By back-projecting the marker position along the projection axis of the imaging system for two images from different points of view, the three-dimensional position of the marker may be reconstructed at the crossing of both axes.

After conversion of the beam directions, target points, and marker positions from the treatment plan, where they are stored in the common LINAC geometry (two rotational degrees of freedom for the beam, one rotational degree of freedom for the patient), to the three rotational degrees of freedom of the kappagoniometer at ID17, the actual positions of the markers are compared to the desired position from the TPS. Here, a quaternion-based algorithm [88] computes the best translation and rotation, which minimize the squared distance between actual positions and desired position. After application of this transform to the sample stage, an additional projection image form beam's eye view is taken and overlaid with information from the treatment plan (e.g. desired marker position, target point, target volume), thus offering an easily human-readable verification of proper alignment.

The imaging of the patient is done using the ID17 wigglerinduced X-ray beam ( $25 \mathrm{~mm}$ wide, $0.2 \mathrm{~mm}$ high), which require scanning the patient vertically through the beam while acquiring a sequence of projection images and repeating the procedure for several columns. The resulting data is stitched together adequately to get a projection image whose maximum dimensions are limited to $13 \mathrm{~cm} \times 13 \mathrm{~cm}$ by the ranges of the motors involved.

The imaging dose has been measured with two different ionization chambers, the PTW Semiflex 31,010 chamber with $0.125 \mathrm{~cm}^{3}$ sensitive volume and the Radcal 10X6-6 detector with $6 \mathrm{~cm}^{3}$ sensitive volume. Since the X-ray beam does not have a homogeneous intensity at the wiggler gap used for imaging, the detector reading has been corrected with a factor generated by numerically integrating the inhomogeneous beam intensity profile over the sensitive detector volume, revealing an effect of approximately $2 \%$ on the Semiflex detector and $15 \%$ on the Radcal detector. Furthermore, 
corrections for different calibration conditions and beam qualities were applied. The resulting entrance surface air kerma ranges between $3.5 \mathrm{mGy}$ and $12 \mathrm{mGy}$ depending on the beam intensity chosen. In all configurations the lead markers were clearly visible on radiographs taken of an anthropomorphic head phantom, but for low doses fine details were covered by detector noise. Comparing these findings to diagnostic reference levels for human head radiographs [89], which are quoted as $3 \mathrm{mGy}$ for lateral and $5 \mathrm{mGy}$ (entrance surface air kerma) for anterior-posterior skull imaging, proves the safety of the imaging procedure.

Several tests of the imaging procedure have been performed with the same anthropomorphic head phantom to benchmark the precision of two different protocols: A first series of irradiations using four lead markers ( $1.7 \mathrm{~mm}$ diameter), which were removed after CT imaging and placed again on the phantom for irradiation, and a second series using four smaller titanium markers $(\sim 1.0 \mathrm{~mm}$ diameter), which were not removed from the phantom. The former protocol was meant to stay as close as possible to the procedure that will be applied in upcoming veterinary trials, while the latter focusing on maximum precision from the technical point of view. A total of six treatment plans of the first protocol and two treatment plans of the second protocol were applied, while radiochromic film placed at the target point inside the phantom recorded the actual point that was irradiated. Conformal irradiations were carried out for the second protocol to show the feasibility of this technique.

The maximum deviation from the targeted point measured for the first protocol was $1.65 \mathrm{~mm}$, while the maximum deviation for the second protocol was of $0.57 \mathrm{~mm}$. Additionally the angular misalignment was measured for the first protocol, which gave a maximum deviation of $1.47^{\circ}$. The alignment of the conformal mask has shown to be accurate. The installation of a synchronized slave motor for the separate movement of the conformal mask is foreseen to prevent vibrations of the mask during the movement, which has been revealed by these experiments that used a lever arm for synchronization.

With the development of image-guided conformal MRT, the ESRF biomedical beamline ID17 is technically ready for the irradiation of deep-seated tumors of cats and dogs in first veterinary trials. The full chain from treatment planning to precise irradiation including conformal masks has been tested and works properly. A graphical user interface for easy control of the image-guidance software is currently under development.

\section{Discussion and conclusions}

With the currently ongoing SSRT clinical trials it will be possible to refine a protocol for dose enhancement using high $\mathrm{Z}$ elements in combination with low-energy SR X-rays. Such an improved protocol can on one hand possibly be exploited at the clinics using higher photon energies and on the other hand be combined later with clinical trials in MRT with a refined protocol for drug delivery to combine the synergetic effects in one single novel radiation therapy approach.

With the veterinary trials currently underway in MRT, the treatment of deep seated tumors with spatially fractionated low energy photons in pets is challenging due to the presence of thick bone structures. The gain from conformal image guided MRT treatment in the near future using 3 multiple ports will provide an initial first geometrical improvement, which can soon be handled from a technical and safety point of view. Further steps may be necessary to increase the TCP while reducing possible normal tissue complications. With increasingly complex irradiation modalities, the challenges on high precision dose measurements within microscopically small movements represent an important step to define a safe treatment strategy with optimized dose delivery and a high therapeutic index. It should be kept in mind that the demands on the Medical Physics developments may be beyond the current technology available and potential additional uncertainties in dose delivery can be handled by a lower peak entrance dose to cope with the lacking accuracy to make the treatment safe. The therapeutic gain using spatially fractionated beams to exploit the dose volume effect and efficiently reduce tumor volume by introducing a preferential damage on the tumor vasculature should nevertheless be visible, even when peak entrance dose values include additional safety margins.

A number of other applications using microbeams are now emerging, including the treatment of epilepsy, Parkinson's disease or chronic pain, and it is worth pointing out that the Medical Physics aspects relating to dosimetry and the design of the TPS are identical for these studies, too. In the presence of respiratory motion and cardiac pulsatility, it may perhaps never be possible to achieve the precision required for the complete joining of interlaced microbeams in larger targets, as would be needed to translate some of the more advanced approaches to treat humans safely. Nevertheless, there is much scope for important technical developments in the years to come, whilst, at present, the more exotic applications are instead using the microbeams as a research tool to improve our understanding of the underlying mechanisms. Similar considerations apply to the applications of larger "microbeams", often also referred to as minibeams, but in this case, much of the biological data is, as yet, largely missing. Over the past 25 years a vast amount of experience in MRT, together with preclinical data, have been accumulated and for this reason we believe that we are justified in our claim that MRT has matured into a technique to be promoted soon for clinical trials.

\section{Acknowledgement}

We wish to acknowledge the support of the currently ongoing COST action SYRA3 (COST Action TD1205), which has allowed to foster this collaborative network and in particular the possibility of the Short Term Scientific Missions permitting an efficient exchange of staff to perform common experiments and improve communication.

\section{References}

[1] Biston MC, Joubert A, Adam JF, Elleaume H, Bohic S, Charvet AM, et al. Cure of Fisher rats bearing radioresistant F98 glioma treated with cis-platinum and irradiated with monochromatic synchrotron X-rays. Cancer Res 2004;64(7): 2317-23.

[2] Adam JF, Joubert A, Biston MC, Charvet AM, Peoc'h M, Le Bas JF, et al. Prolonged survival of fischer rats bearing F98 glioma after iodine-enhanced synchrotron stereotactic radiotherapy. Int J Radiat Oncol Biol Phys 2006;64(2):603-11.

[3] Rose JH, Norman A, Ingram M, Aoki C, Solberg T, Mesa A. First radiotherapy of human metastatic brain tumors delivered by a computerized tomography scanner (CTRx). Int J Radiat Oncol Biol Phys 1999;45(5):1127-32.

[4] Renier M, Brochard T, Nemoz C, Requardt H, Bräuer E, Esteve F, et al. The radiotherapy clinical trials projects at the ESRF: technical aspects. Eur J Radiol Dec. 2008:68(Suppl. 3):S147-50.

[5] Vautrin M. Planification de traitement en radiothérapie stéréotaxique par rayonnement synchrotron. Développement et validation d'un module de calcul de dose par simulations Monte Carlo. Université de Grenoble; 2011.

[6] Prezado Y, Vautrin M, Martiínez-Rovira I, Bravin A, Estève F, Elleaume H, et al. Dosimetry protocol for the forthcoming clinical trials in synchrotron stereotactic radiation therapy (SSRT). Med Phys 2011:38(3):1709-17.

[7] Obeid L, Deman P, Tessier A, Balosso J, Estève F, Adam J-F. Absolute perfusion measurements and associated iodinated contrast agent time course in brain metastasis: a study for contrast-enhanced radiotherapy. J Cereb Blood Flow Metab Apr. 2014;34(4):638-45.

[8] Balosso J, Estève F, Elleaume H, Bravin A, Adam JF, Renier M, et al. Monoenergetic synchrotron beams: first human experience for therapeutic purpose. Vienna, Austria: ESTRO 33; 2014. SP-0205.

[9] Dilmanian FA, Zhong Z, Bacarian T, Benveniste H, Romanelli P, Wang R, et al. Interlaced $\mathrm{X}$-ray microplanar beams: a radiosurgery approach with clinical potential. Proc Natl Acad Sci U. S. A Jun. 2006;103(25):9709-14. 
[10] Spasic E, Adam J-F. Optically stimulated luminescence for diagnostic and therapeutic low to medium energy X-ray beams experimental dosimetry. Phys Medica Jun. 2013;29:e42.

[11] Le Bourhis J, Vautrin M, Elleaume H, Esteve F, Desbat L, Nemoz C, et al. Local and limited projection tomography reconstructions for contrast-enhanced synchrotron stereotactic radiotherapy in vivo dosimetry. Med Phys Jul. 2011;38(6):3534.

[12] Deman P, Vautrin M, Edouard M, Stupar V, Bobyk L, Farion R, et al. Monochromatic minibeams radiotherapy: from healthy tissue-sparing effect studies toward first experimental glioma bearing rats therapy. Int J Radiat Oncol Biol Phys Mar. 2012;82(4):e693-700.

[13] Prezado Y, Fois G, Edouard M, Nemoz C, Renier M, Requardt H, et al. Biological equivalent dose studies for dose escalation in the stereotactic synchrotron radiation therapy clinical trials. Med Phys 2009;36(3):725-33.

[14] Bobyk L, Edouard M, Deman P, Vautrin M, Pernet-Gallay K, Delaroche J, et al. Photoactivation of gold nanoparticles for glioma treatment. Nanomedicine Oct. 2013;9(7):1089-97.

[15] Rousseau J, Barth RF, Moeschberger ML, Elleaume H. Efficacy of intracerebral delivery of carboplatin in combination with photon irradiation for treatment of F98 glioma-bearing rats. Int J Radiat Oncol Biol Phys 2009;73(2):530-6.

[16] Slatkin DN, Spanne P, Dilmanian FA, Sandborg M. Microbeam radiation therapy. Med Phys 1992;19(6):1395-400.

[17] Archer DW. Collimator for producing an array of microbeams. 1998.

[18] Laissue JA, Lyubimova N, Wagner H-P, Archer DW, Slatkin DN, Di Michiel M, et al. Microbeam radiation therapy. In: SPIE's international symposium on optical science, engineering, and instrumentation; 1999. p. 38-45.

[19] Laissue JA, Blattmann H, Di Michiel M, Slatkina DN, Lyubimovaa N, Guzmand R, et al. The weanling piglet cerebellum : a surrogate for tolerance to MRT (microbeam radiation therapy) in pediatric neuro-oncology. Proc SPIE 2001;4508:65-73.

[20] Stepanek J, Blattmann H, Laissue JA, Lyubimova N, Di Michiel M, Slatkin DN Physics study of microbeam radiation therapy with PSI-version of Monte carlo code GEANT as a new computational tool. Med Phys 2000;27(7): $1664-75$.

[21] Siegbahn EA, Stepanek J, Bräuer-Krisch E, Bravin A. "Determination of dosimetrical quantities used in microbeam radiation therapy (MRT) with Monte carlo simulations. Med Phys 2006:33(9):3248.

[22] Hugtenburg RP, Adegunloye AS, Bradley DA. X-ray microbeam radiation therapy calculations, including polarisation effects, with the Monte carlo code EGS5. Nucl Instrum Meth A 2010;619(1-3):221-4.

[23] De Felici M, Felici R, Sanchez del Rio M, Ferrero C, Bacarian T, Dilmanian FA. Dose distribution from $\mathrm{x}$-ray microbeam arrays applied to radiation therapy: an EGS4 Monte carlo study. Med Phys 2005;32(8):2455-63.

[24] Martínez-Rovira I, Sempau J, Prezado Y. Development and commissioning of a Monte carlo photon beam model for the forthcoming clinical trials in microbeam radiation therapy. Med Phys Jan. 2012;39(1):119-31.

[25] Bartzsch S, Lerch M, Petasecca M, Bräuer-Krisch E, Oelfke U. Influence of polarization and a source model for dose calculation in MRT. Med Phys 2014;41(4):041703.

[26] Bartzsch S, Oelfke U. A new concept of pencil beam dose calculation for 40-200 keV photons using analytical dose kernels. Med Phys Nov. 2013:40(11):111714.

[27] Griffin RJ, Koonce NA, Dings RPM, Siegel E, Moros EG, Bräuer-Krisch E, et al. Microbeam radiation therapy alters vascular architecture and tumor oxygenation and is enhanced by a galectin- 1 targeted anti-angiogenic peptide. Radiat Res Jun. 2012;177(6):804-12.

[28] Uyama A, Kondoh T, Nariyama N, Umetani K, Fukumoto M, Shinohara K, et al. A narrow microbeam is more effective for tumor growth suppression than a wide microbeam: an in vivo study using implanted human glioma cells. J Synchrotron Radiat Jul. 2011;18(Pt 4):671-8.

[29] Martínez-Rovira I, Sempau J, Fernández-Varea JM, Bravin A, Prezado Y. Monte carlo dosimetry for forthcoming clinical trials in X-ray microbeam radiation therapy. Phys Med Biol Aug. 2010;55(15):4375-88.

[30] Bräuer-Krisch E, Requardt H, Régnard P, Corde S, Siegbahn E, Le Duc G, et al. New irradiation geometry for microbeam radiation therapy. Phys Med Biol Jul. 2005;50(13):3103-11.

[31] Anderson D, Siegbahn EA, Fallone BG, Serduc R, Warkentin B. Evaluation of dose-volume metrics for microbeam radiation therapy dose distributions in head phantoms of various sizes using Monte carlo simulations. Phys Med Biol May 2012;57(10):3223-48.

[32] Andreo P, Izewska J, Shortt K, Vatnitsky S. IAEA TRS 430-Commissioning and quality assurance of computerized treatment planning systems for radiation treatment of Cancer. Vienna: International Atomic Energy Agency; 2004.

[33] Bräuer-Krisch E, Bravin A, Siegbahn E, Zhang AL, Stepanek J, Blattmann H, et al. Characterization of a tungsten/gas multislit collimator for microbeam radiation therapy at the European synchrotron radiation facility. Rev Sci Instrum 2005;76:1-7.

[34] Bräuer-Krisch E, Rosenfeld A, Lerch M, Petasecca M, Akselrod M, Sykora J, et al. Potential high resolution dosimeters for MRT. AIP Conf Proc 2010;1266: 89-97.

[35] Crosbie JC, Svalbe I, Midgley SM, Yagi N, Rogers PA, Lewis RA. A method of dosimetry for synchrotron microbeam radiation therapy using radiochromic films of different sensitivity. Phys Med Biol 2008:53(23):6861-77.

[36] Akselrod GM, Akselrod MS, Benton ER, Yasuda N. "A novel Al2O3 fluorescent nuclear track detector for heavy charged particles and neutrons. Nucl Instrum
Methods Phys Res Sect B Beam Interact Mater Atoms Jun. 2006;247(2): 295-306.

[37] Sykora GJ, Akselrod MS. Novel fluorescent nuclear track detector technology for mixed neutron-gamma fields. Radiat Meas Mar. 2010;45(3-6):594-8.

[38] Ptaszkiewicz M, Brauer-Krisch E, Klosowski M, Czopyk L, Olko P. TLD dosimetry for microbeam radiation therapy at the European synchrotron radiation facility. Radiat Meas 2008;43:990-3.

[39] Petasecca M, Cullen A, Fuduli I, Espinoza A, Porumb C, Stanton C, et al. Xtream: a novel dosimetry system for synchrotron microbeam radiation therapy. J Instrum 2012;7:P07022.

[40] Rosenfeld AB, Lerch MLF, Kron T, Brauer-Krisch E, Bravin A, Holmes-Siedle A, et al. Feasibility study of online high-spatial-resolution MOSFET dosimetry in static and pulsed X-ray radiation fields. IEEE Trans Nucl Sci 2001;48(6): 2061-8.

[41] Bräuer-Krisch E, Bravin A, Lerch M, Rosenfeld A, Stepanek J, Di Michiel M, et al MOSFET dosimetry for microbeam radiation therapy at the European synchrotron radiation facility. Med Phys Apr. 2003;30(4):583-9.

[42] Lerch MLF, Petasecca M, Cullen A, Hamad A, Requardt H, et al. Dosimetry of intensive synchrotron microbeams. Radiat Meas Dec. 2011;46(12):1560-5.

[43] Okada Go, Vahedi Shahatzad, Morrell Brian, Koughia Cyril, Belev George, Wysokinski Tomasz, et al. Examination of the dynamic range of Sm-doped glasses for high-dose and high-resolution dosimetric applications in microbeam radiation therapy at the Canadian synchrotron. Opt Mater 01/ 2012;11(35):1976-80.

[44] Vahedi Shahatzad, Okada Go, Morrell Brian, Muzar Edward, Koughia Cyril Edgar Andy, et al. X-ray induced $\mathrm{SM} 3+$ to $\mathrm{Sm} 2+$ conversion in fluorophosphate and fluoroaluminate glasses for the monitoring of high-doses in microbeam radiation therapy. J Appl Phys 01/2012;112:073108.

[45] Okada Go, Morrell Brian, Koughia Cyril, Edgar Andy, Varoy Chirs, Belev George, Wysokinski Tomasz, Chapman Dean, Kasap Safa. Spatially resolved measurement of high doses in microbeam radiation therapy using samarium doped fuorophosphatte glasses. Appl Phys Lett 01/2011;99 (Comendex).

[46] Sporea D, Mihai L, Sporea A, Lixandru A, Bräuer-Krisch E. Investigation of UV optical fibers under synchrotron irradiation. Opt Express Dec. 2014;22(25): 31473.

[47] Rink A, Lewis DF, Varma S, Vitkin IA, Jaffray DA. Temperature and hydration effects on absorbance spectra and radiation sensitivity of a radiochromic medium. Med Phys Oct. 2008;35(10):4545-55.

[48] Koulouklidis AD, Cohen S, Kalef-Ezra J. Thermochromic phase-transitions of GafChromic films studied by z-scan and temperature-dependent absorbance measurements. Med Phys Nov. 2013:40(11):112701.

[49] Niroomand-Rad A, Blackwell CR, Coursey BM, Gall KP, Galvin JM, McLaughlin WL, et al. Radiochromic film dosimetry: recommendations of AAPM radiation therapy committee task group 55. American association of physicists in medicine. Med Phys Nov. 1998;25(11):2093-115.

[50] Soares CG. Radiochromic film dosimetry. Radiat Meas Dec. 2006;41:S100-16.

[51] Devic S. Radiochromic film dosimetry: past, present, and future. Phys Med Jul. 2011:27(3):122-34.

[52] Kalef-Ezra J, Karava K. Radiochromic film dosimetry: reflection vs transmission scanning. Med Phys Jun. 2008;35(6):2308-11.

[53] Micke A, Lewis DF, Yu X. Multichannel film dosimetry with nonuniformity correction. Med Phys 2011;38(5):2523.

[54] Lewis D, Micke A, Yu X, Chan MF. An efficient protocol for radiochromic film dosimetry combining calibration and measurement in a single scan. Med Phys Oct. 2012;39(10):6339-50.

[55] Siegbahn EA, Bräuer-Krisch E, Bravin A, Nettelbeck H, Lerch MLF, Rosenfeld AB. MOSFET dosimetry with high spatial resolution in intense synchrotron-generated x-ray microbeams. Med Phys Apr. 2009;36(4): $1128-37$.

[56] Bartzsch S. Microbeam radiation therapy - physical and biological aspects of a new cancer therapy and development of a treatment planning system. University of Heidelberg; 2014.

[57] Hermida-López M, Lüdemann L, Flühs A, Brualla L. Technical Note: Influence of the phantom material on the absorbed-dose energy dependence of the EBT3 radiochromic film for photons in the energy range $3 \mathrm{keV}-18 \mathrm{MeV}$. Med Phys Nov. 2014:41(11):112103.

[58] Muench PJ, Meigooni AS, Nath R, McLaughlin WL. Photon energy dependence of the sensitivity of radiochromic film and comparison with silver halide film and LiF TLDs used for brachytherapy dosimetry. Med Phys 1991;18(4): $769-75$.

[59] Kron T, Duggan L, Smith T, Rosenfeld A, Butson M, Kaplan G, et al. Dose response of various radiation detectors to synchrotron radiation. Phys Med Biol Nov. 1998;43(11):3235-59.

[60] Nariyama N. Responses of GafChromic films for distribution of extremely high doses from synchrotron radiation. Appl Radiat Isot May 2005;62(5) 693-7.

[61] Cheung T, Butson MJ, Yu PKN. Experimental energy response verification of XR type T radiochromic film. Phys Med Biol Nov. 2004;49(no. 21): N371-6.

[62] Oves SD, Hogstrom KR, Ham K, Sajo E, Dugas JP. Dosimetry intercomparison using a 35-keV X-ray synchrotron beam. Eur J Radiol Dec. 2008;68(3 Suppl): S121-5.

[63] Brown TAD, Hogstrom KR, Alvarez D, Matthews KL, Ham K, et al. Doseresponse curve of EBT, EBT2, and EBT3 radiochromic films to synchrotronproduced monochromatic X-ray beams. Med Phys Dec. 2012;39(12):7412-7. 
[64] Gill S, Hill R. A study on the use of Gafchromic ${ }^{\text {TM }}$ EBT3 film for output factor measurements in kilovoltage X-ray beams. Australas Phys Eng Sci Med Dec. 2013;36(4):465-71.

[65] Villarreal-Barajas JE, Khan RFH. Energy response of EBT3 radiochromic films: implications for dosimetry in kilovoltage range. J Appl Clin Med Phys Jan 2014;15(1):4439.

[66] Massillon-JL G. Energy dependence of the new gafchromic EBT3 Film: dose response curves for 50 KV, 6 and 15 MV X-ray beams. Int J Med Phys Clin Eng Radiat Oncol Aug. 2012;01(02):60-5.

[67] A. Appleby, E. A. Christman, and A. Leghrouz, Imaging of spatial radiation dose distribution in agarose gels using magnetic resonance, Med Phys. 14. 3, pp. $382-384$.

[68] Schreiner LJ. Review of fricke gel dosimeters. J Phys Conf Ser Jan. 2004;3(1): 9-21.

[69] Maryanski MJ, Gore JC, Kennan RP, Schulz RJ. NMR relaxation enhancement in gels polymerized and cross-linked by ionizing radiation: a new approach to 3D dosimetry by MRI. Magn Reson Imaging Jan. 1993;11(2):253-8.

[70] Gore JC, Ranade M, Maryañski MJ, Schulz RJ. Radiation dose distributions in three dimensions from tomographic optical density scanning of polymer gels: I. Development of an optical scanner. Phys Med Biol Dec. 1996;41(12): 2695-704.

[71] Doran SJ, Koerkamp KK, Bero MA, Jenneson P, Morton EJ, Gilboy WB. A CCDbased optical CT scanner for high-resolution 3D imaging of radiation dose distributions: equipment specifications, optical simulations and preliminary results. Phys Med Biol Dec. 2001;46(12):3191-213.

[72] Boudou C, Troprès I, Rousseau J, Lamalle L, Adam JF, Estève F, et al. Polymer gel dosimetry for synchrotron stereotactic radiotherapy and iodine doseenhancement measurements. Phys Med Biol Aug. 2007;52(16):4881-92.

[73] Berg A, Pernkopf M, Waldhäusl C, Schmidt W, Moser E. High resolution MR based polymer dosimetry versus film densitometry: a systematic study based on the modulation transfer function approach. Phys Med Biol Sep. 2004;49(17):4087-108.

[74] Bayreder C, Schön R, Wieland M, Georg D, Moser E, Berg A. The spatial resolution in dosimetry with normoxic polymer-gels investigated with the dose modulation transfer approach. Med Phys May 2008;35(5):1756-69.

[75] Adamovics J, Maryanski MJ. Characterisation of PRESAGE: a new 3-D radiochromic solid polymer dosemeter for ionising radiation. Radiat Prot Dosim Jan. 2006;120(1-4):107-12.
[76] Skyt PS, Balling P, Petersen JBB, Yates ES, Muren LP. Temperature dependence of the dose response for a solid-state radiochromic dosimeter during irradiation and storage. Med Phys May 2011;38(5):2806-11.

[77] Skyt PS, Wahlstedt I, Muren LP, Petersen JBB, Balling P. Temperature and temporal dependence of the optical response for a radiochromic dosimeter. Med Phys Dec. 2012;39(12):7232-6.

[78] Al Nowais S, Kacperek A, Brunt JNH, Adamovics J, Nisbet A, Doran SJ. An investigation of the response of the radiochromic dosimeter PRESAGE TM to irradiation by $62 \mathrm{MeV}$ protons. J Phys Conf Ser Nov. 2010;250(1):012034.

[79] Annabell N, Yagi N, Umetani K, Wong C, Geso M. Evaluating the peak-to-valley dose ratio of synchrotron microbeams using PRESAGE fluorescence. J Synchrotron Radiat May 2012;19(Pt 3):332-9.

[80] Doran SJ, Abdul Rahman AT, Bräuer-Krisch E, Brochard T, Adamovics J, Nisbet A, et al. Establishing the suitability of quantitative optical CT microscopy of PRESAGE ${ }^{\circledR}$ radiochromic dosimeters for the verification of synchrotron microbeam therapy. Phys Med Biol Sep. 2013;58(18):6279-97.

[81] Doran SJ, Brochard T, Adamovics J, Krstajic N, Bräuer-Krisch E. An investigation of the potential of optical computed tomography for imaging of synchrotron-generated X-rays at high spatial resolution. Phys Med Biol Mar. 2010;55(5):1531-47.

[82] Serduc R, Bräuer-Krisch E, Siegbahn EA, Bouchet A, Pouyatos B, Carron R, et al. High-precision radiosurgical dose delivery by interlaced microbeam arrays of high-flux low-energy synchrotron X-rays. PLoS One 2010;5(2):12.

[83] Donzelli M. Implementation of conformal image-guided Microbeam radiation Therapy for veterinary Trials at the ESRF biomedical beamline ID17. RuprechtKarls-Universität Heidelberg, Ruprecht-Karls-Universität Heidelberg. 2014.

[84] Doran SJ, Rahman ATA, Bräuer-Krisch E, Brochard T, Adamovics J. Ultra-high resolution optical CT dosimetry for the visualisation of synchrotron microbeam therapy doses. J Phys Conf Ser Jun. 2013;444(1):012074.

[85] Low DA, Harms WB, Mutic S, Purdy JA. A technique for the quantitative evaluation of dose distributions. Med Phys May 1998;25(5):656.

[86] 3DMiMic collaboration No Title. [Online]. Available:www.sintef.no/3dmimic

[87] Bendl R, Hoess A, Schlegel W. Virtual simulation in radiotherapy planning. In: computer vision, virtual reality and robotics in medicine: 1995. p. 287-92.

[88] Horn BKP. Closed-form solution of absolute orientation using unit quaternions. J Opt Soc Am A 1987;4(4):629-42.

[89] Diagnostic reference levels in medical imaging: review and additional advice. Ann ICRP Jan. 2001;31(4):33-52. 